\title{
Chapter 11 \\ Bond Graph Modeling and Simulation of a Vibration Absorber System in Helicopters
}

\author{
Benjamin Boudon, François Malburet, and Jean-Claude Carmona
}

\section{Notation}

Subscripts

$i \quad$ Relative to the $i$ loop $(i=1 \ldots 4)$

$j \quad$ Relative to the body $j\left(j=\mathrm{MGB}, \mathrm{SB}_{i}, \mathrm{MB}_{i}, F\right)$

MGB Relative to the body MGB

$\mathrm{SB}_{i} \quad$ Relative to the body SARIB bar $i$

$\mathrm{MB}_{i} \quad$ Relative to the body MGB bar $i$

$F \quad$ Relative to the body fuselage

\section{Mechanical Notation}

\section{Generality}

$\overrightarrow{M N}^{h}$
$\vec{P}_{\text {pes } \rightarrow j}$ Vector associated with the bipoint (MN) expressed in the $R_{h}$ frame Weight vector of body $j$ expressed in the inertial reference frame $R_{0}$

B. Boudon ( $\square)$

Aix-Marseille Université, CNRS, ISM UMR 7287,

13288, Marseille Cedex 09, France

e-mail: benjamin.boudon@univ-amu.fr

F. Malburet $\bullet$ J.-C. Carmona

Arts-et-Métiers ParisTech, CNRS, LSIS, 2 Cours des Arts-et-Métiers,

13617, Aix-en-Provence, France 
$\vec{\Omega}(j / 0)^{j} \quad$ Angular velocity vector of body $j$ with regard to the inertial reference frame expressed in the frame $R_{j}$

$\vec{V}\left(M / R_{0}\right)^{j} \quad$ Linear absolute velocity (in regard to the inertial frame) of point $M$ expressed in the frame $R_{j}$

$\vec{V}(M \in j / k)^{j} \quad$ Linear relative velocity vector of point $M$ of body $j$ with regard to the body k expressed in the frame $R_{j}$

$P_{0}^{j} \quad$ Transformation matrix from the inertial reference frame $R_{0}$ to the frame $R_{j}$

$\tau\left(\right.$ ext $\left.\rightarrow S_{j}\right) \quad$ External mechanical wrench applied to body $j$

\section{Model Minutiae}

$G_{j} \quad$ Body $j$ 's center of mass

$O_{\mathrm{FF}} \quad$ Origin of fixed frame

$A_{i} \quad$ Point located on the revolute joint between the fuselage and the SARIB beater $i$

$B_{i} \quad$ Center of the spherical joint between an SARIB beater $i$ and a MGB bar $i$

$C_{i} \quad$ Center of the spherical joint between an MGB bar $i$ and the MGB

$A_{\mathrm{MGB}}$ Point located on the MGB on the prismatic joint with the fixed frame

\section{Reference Frames}

$$
\begin{array}{ll}
R_{0}=\left(O_{0}, \vec{x}_{0}, \vec{y}_{0}, \vec{z}_{0}\right) & \begin{array}{l}
\text { Inertial reference frame (or Galilean } \\
\text { frame) }
\end{array} \\
R_{j}=\left(O_{j}, \vec{x}_{j}, \vec{y}_{j}, \vec{z}_{j}\right) & \begin{array}{l}
\text { Local reference frame of body } j \\
\text { Local reference frame }\left(R_{\mathrm{FF}}=R_{0}\right) \\
R_{\mathrm{FF}}=\left(O_{\mathrm{FF}}, \vec{x}_{\mathrm{FF}}, \vec{y}_{\mathrm{FF}}, \vec{z}_{\mathrm{FF}}\right)
\end{array} \\
R_{\mathrm{MGB}}=\left(G_{\mathrm{MGB}}, \vec{x}_{\mathrm{MGB}}, \vec{y}_{\mathrm{MGB}}, \vec{z}_{\mathrm{MGB}}\right) & \begin{array}{l}
\text { attached to the fixed frame } \\
\text { Local reference frame attached to the } \\
\text { MGB }
\end{array} \\
R_{F}=\left(G_{F}, \vec{x}_{F}, \vec{y}_{F}, \vec{z}_{F}\right) & \begin{array}{l}
\text { Local reference frame attached to the } \\
\text { fuselage }
\end{array} \\
R_{F} \text { int }_{i}=\left(A_{i}, \vec{x}_{F} \text { int }_{i}, \vec{y}_{F} \text { int }_{i}, \vec{z}_{F} \text { int }_{i}\right) & \begin{array}{l}
\text { Intermediate local reference frame } \\
\text { attached to fuselage so as to facilitate }
\end{array} \\
& \text { the definition of the axis of the revolute } \\
\text { joints } & \text { Local reference frame attached to the } \\
R_{\mathrm{SB}}=\left(G_{\mathrm{SB}}, \vec{x}_{\mathrm{SB}}, \vec{y}_{\mathrm{SB}}, \vec{z}_{\mathrm{SB}}\right) & \text { SARIB Beaters } \\
R_{\mathrm{MB}}=\left(G_{\mathrm{MB}}, \vec{x}_{\mathrm{MB}}, \vec{y}_{\mathrm{MB}}, \vec{z}_{\mathrm{MB}}\right) & \begin{array}{l}
\text { Local reference frame attached to the } \\
\text { MGB Bar }
\end{array}
\end{array}
$$




\section{Model Variables}

$x_{j}, y_{j}, z_{j} \quad$ Positional parameters of body $j$ 's center of mass (m)

$\alpha_{j}, \beta_{j}, \gamma_{j} \quad$ Angular parameters of the body $j$ with regard to the inertial reference frame (rad)

\section{Model Parameters}

$\Omega \quad$ Rotational velocity of the main rotor $\left(\operatorname{rad} . \mathrm{s}^{-1}\right)$

$b \quad$ Number of rotor's blades (adim)

$m_{j} \quad$ Body j's mass $(\mathrm{kg})$

$I_{G j} \quad$ Body $j$ 's Inertia matrix $\left(\mathrm{kg} \mathrm{m}^{2}\right)$

\subsection{Introduction}

In the 1990s, thanks to the multibond graph formalism (an extension of bond graphs where the scalar power bonds become vectors bonds and the elements multiports), the bond graph was extended to the study of multibody systems with three dimensions [7, 29]. Nevertheless, few complex multibody systems with kinematic closed loops have been simulated. In the last 20 years, computer science and software dedicated to bond graphs such as 20-sim software have progressed considerably and contributed greatly to the development of bond graphs [1]. First, the graphical aspect of bond graphs can be fully exploited. Indeed, friendly environments enable the entering, modifying, or interpreting of bond graphs. Secondly, the automatic generation of equations from a bond graph enables engineers to avoid solving equations. Consequently, this step is less cumbersome and prone to errors. Moreover, the simulation software mentioned is now equipped with efficient numerical solvers.

This chapter presents a bond graph model and the associated simulations of a complex multibody system: a vibration absorber system in helicopters. The system model is a complex multibody system (according to the definitions given in [28]) because of the numerous bodies and joints and the presence of several kinematic loops.

The operation of a helicopter's rotor causes important vibration levels affecting, namely the flight handling, the fatigue of the mechanical parts, and the crew's comfort. The considered vibrations created by the aerodynamic and inertia forces acting on the rotor excite the main gearbox then the fuselage at a specific frequency $b \Omega$ where $b$ is the number of blades of the main rotor and $\Omega$ the rotational velocity of the main rotor. Suspensions between the main gear box (MGB) and the fuselage help to filter these problematic vibrations. Different passive technical solutions exist 
for the completion of this joint. The role of the studied vibration absorber system is to reduce the efforts transmitted from the rotor to the fuselage at the $b \Omega$ frequency by creating an anti-resonance phenomenon. The principle of this system will be described in Sect. 11.4.

The design and the analysis of such complex systems are usually conducted with analytical methods based on physical equations or signal-flow methods based on transfer functions written with block diagrams form. Unfortunately, these two classical approaches may cause a loss of the physical sense and the visibility of the modeling assumptions [2,33]. Moreover, reusing models and taking into account the increasing complexity can be cumbersome and prone to errors because of the need of manual transformations so as to build physics-based model libraries with block diagrams [33]. In this context, we present a bond graph (BG) approach [23] that permits a structural and modular approach of a complex mechatronic system. These well-known features [5, 27]: graphic, object-oriented, multiphysic, and acausal can be exploited for this class of multibody system with embedded electronics. Mainly with its graphical nature, the bond graph brings a more global view and comprehension of complex mechatronic systems which lead to more sustainable solutions. Based on oriented-object and acausal features, bond graphs also permit a modular approach which allows a better knowledge capitalization to better store and reuse your modeling works. Moreover, it would facilitate the automation of the modeling task.

The objective of this chapter is to model and simulate a helicopter's vibration absorber system with bond graphs. Particular attention will be given in showing the interest of the bond graph tool relative to more conventional tools.

This chapter is organized as follows. In Sect. 11.2, we review the modeling and simulation methods of multibody systems based on a bond graph approach. Section 11.3 is dedicated to the presentation of the vibration absorber system. Section 11.4 starts by describing the kinematic structure and the mechanical assumptions retained for the suspension model. After, the modeling and simulation framework is applied and the construction of the bond graph model of the vibration absorber system detailed. Simulation results will be presented in Sect. 11.5. Finally, in the last section, the conclusion will be given.

\subsection{BG Modeling and Simulation of Multibody Systems}

\subsubsection{Brief Review on the BG Modeling of Multibody Systems}

The aim of this section is to recall the main contributors concerning methodologies for modeling the dynamics of three-dimensional multibody systems (MBS). More detailed reviews specifying applications of BG modeling for MBS can be found in $[5,13,27]$. 
The first bond graph models of multibody systems have been proposed by D.C. Karnopp and R.C. Rosenberg [16, 26], thanks to an analytical approach based on an appropriate choice of generalized coordinates, the construction of junction structure for the formulation of the kinematic laws, and a Lagrangian formulation.

In the 1990s, thanks to the multibond graph (MBG) formalism [4, 9] (an extension of bond graphs where the scalar power bonds become vector bonds and the elements multiports), the application fields of the bond graph were extended to the study of multibody systems with three-dimensional movements.

The bond graph approach used for multibody systems was introduced by A. M. Bos [7, 29]. In his PhD, he developed bond graph models for three-dimensional multibody systems and discussed how to derive the equations of motion from the bond graph in several forms. At this time, although he managed to conduct simulations of a $3 \mathrm{D}$ motorcycle, the equations had been derived with a manual process.

Library models for a rigid body and for various types of joints have been provided in [34] so that bond graph models of rigid multibody systems can be assembled in a systematic manner.

Felez [14] developed a software that helps with modeling multibody systems using bond graphs. To handle derivative causalities with this software, he proposes a way to introduce Lagrange multipliers into the system so as to eliminate derivative causality.

In [30, 31], different methods for simulating BG models have been presented. These simulations have been conducted with a predecessor of 20-sim software and numerically compared between themselves mainly between the computing time and accuracy. Even if the possibility of using multibond graphs was evocated, the difficulty of implementing bond graphs with vector bonds was not mentioned. This point will be detailed in Sect. 11.2.4.

\subsubsection{Approach Chosen: The Tiernego and Bos Method with a 3D Model and Vector Bonds}

Bond graph construction based on multibody dynamic equations can be established either with the Newton-Euler equations or by using Lagrange equations. Depending on the starting point, several bond graph construction methods have been developed:

- the "Tiernego and Bos" method from the application of the Newton-Euler equations,

- the "Karnopp and Rosenberg" method from the application of the Lagrange equations.

In order to keep a modular approach, the more appropriated method for modeling multibody systems with bond graphs is the Bos and Tiernego method [29]. 
This method enables a multibody system to be built in bond graphs as an assembly of bodies and joints. The principle of this method is based on the use of absolute coordinates systems and Newton-Euler equations. Indeed, in such a way, the dynamic equations of a rigid body depend on, obviously, its mass/inertia parameters and also on geometric parameters that are defined only for the considered body. The kinematic joints impose a set of constraints on the effort and flow vectors of the articulation points involved in the assembly of two bodies so that the desired relative motion can be achieved. Consequently, the dynamic equations of the complete system consist of the dynamic equations of each body depending only on its own parameters and the constraint equations of each joint.

From the authors' point of view, a good approach, in the first preliminary design stage and still, in the frame of a modular approach, is to use in a more general way: a 3D model and vector bonds so as to obtain a synthetic model. The use of vector bond graphs (also called multibond graphs) requires multibond graph elements (multibond or vector bond, junctions, multiport energy storage elements, multiport transformers, and gyrators) which are supposed to be known and directly used in this paper. Readers can refer to [13] to find the details of the modeling of those elements.

\subsubsection{Rigid Body Modeling}

Let us remember the architecture of a rigid body multibond graph model based on $[5,7,20,22]$.

This bond graph architecture is based on the Newton-Euler equations (Eqs. (11.1) and (11.2)) with the inertia matrix (modeled with a multiport energy store element $\left[\mathrm{I}_{S_{j}, G_{j}}\right]_{j}$ in the upper part) associated with gyroscopic terms, respectively (modeled with a multiport gyrator element also called Eulerian Junction Structure about mass-center of body $j$ expressed in its frame $\left[\mathrm{EJS}_{G_{j}}\right]_{j}$ and the mass matrix modeled with a multiport energy store element $\left[\mathrm{m}_{j}\right]_{0}$ in the lower part).

$$
\begin{gathered}
\sum_{h} \vec{F}_{h \rightarrow j}^{0}+\vec{P}_{\mathrm{pes} \rightarrow j}^{0}=m_{j} \frac{\mathrm{d}}{\mathrm{d} t}\left(\vec{V}\left(G_{j} / R_{0}\right)^{0}\right)_{0} \\
\sum_{h} \vec{M}_{G}^{j}=\left[I_{G_{j}}\right]^{j}\left[\frac{\mathrm{d}}{\mathrm{d} t}\left(\vec{\Omega}(j / 0)^{j}\right)_{0}\right]^{j}+\vec{\Omega}(j / 0)^{j} \wedge\left(\left[I_{G_{j}}\right]^{j} \vec{\Omega}(j / 0)^{j}\right)
\end{gathered}
$$

The upper part of the bond graph represents the rotational dynamic part expressed in the body frame while the lower part is for the translational dynamic part expressed in the inertial reference frame (or Galilean frame). Notice that the power bonds corresponding to the rotational quantities are in purple while the power bonds corresponding to the translational quantities are in green. The two corresponding 1 -junctions arrays correspond, respectively, to the angular velocity vector of body 
$j$ with regard to the inertial frame $\vec{\Omega}(j / 0)^{j}$ and the translational velocity vector of body j's center of mass in regard to the inertial frame $\vec{V}\left(G_{j} / R_{0}\right)^{0}$ expressed in these two coordinate frames.

The central part of the MBG describes the kinematic relations (Eq. (11.3)) between the velocities of the two points of the body $j\left(\vec{V}\left(M_{k} / R_{0}\right)^{i}\right.$ and $\left.\vec{V}\left(M_{l} / R_{0}\right)^{0}\right)$ and the velocity of the center of mass $\vec{V}\left(G_{j} / R_{0}\right)^{j}$ resulting from the formula of the rigid body.

$$
\begin{aligned}
\vec{V}\left(M_{k} / R_{0}\right)^{0} & =\vec{V}\left(G_{j} / R_{0}\right)^{0}+\vec{\Omega}\left(S_{j} / 0\right)^{0} \wedge{\overrightarrow{G_{j} M_{k}}}^{0} \\
\vec{V}\left(M_{l} / R_{0}\right)^{0} & =\vec{V}\left(G_{j} / R_{0}\right)^{0}+\vec{\Omega}\left(S_{j} / 0\right)^{0} \wedge{\overrightarrow{G_{j} M_{l}}}^{0}
\end{aligned}
$$

As the translational dynamic is expressed in the inertial reference frame, a modulated transformation element (MTF) between $\vec{V}\left(G_{j} / R_{0}\right)^{j}$ and $\vec{V}\left(G_{i} / R_{0}\right)^{0}$ permits the coordinate transformation (Eq. (11.4)) between the body frame $R_{j}$ and the inertial frame $\mathrm{R}_{0}$ (Fig. 11.1).

$$
{\overrightarrow{V\left(G_{j} / R_{0}\right)}}^{0}=P_{0}^{j} \cdot{\overrightarrow{V\left(G_{j} / R_{0}\right)}}^{j}
$$

The rotation matrix $P_{0}^{j}$ can be calculated from Cardan angles. In this paper, the XYZ Cardan angles have been employed. The angular velocity components of the considered body expressed in the body frame (called the pseudo-velocities or Cardan angles rates of changes) are used to determine the body's orientation and the corresponding coordinate transformation matrix. This classical process used in the Cardan block is detailed in Fig. 11.2. It should be noted that the initial conditions used for the integration of time derivatives of the Cardan angles must be consistent with regard to the kinematic constraints.

\subsubsection{Main Difficulties Linked to the Approach Chosen}

\subsubsection{About Causalities Imposed by Vector Bonds}

The first step in carrying out a simulation is the assignment of causalities. Vector bonds create some causality constraints that will force the modeler to choose the right method. Indeed, as we can find in [3, 12], two causality constraints appear with vector bonds.

The first causality constraint $(\mathrm{C} 1)$ is: each component of a vector bond must have the same causality. Consequently, it is not possible to constrain the motion using $\mathrm{Sf}$ elements in only one or two dimensions without introducing some parasitic elements into the remaining unconstrained dimensions(s). 


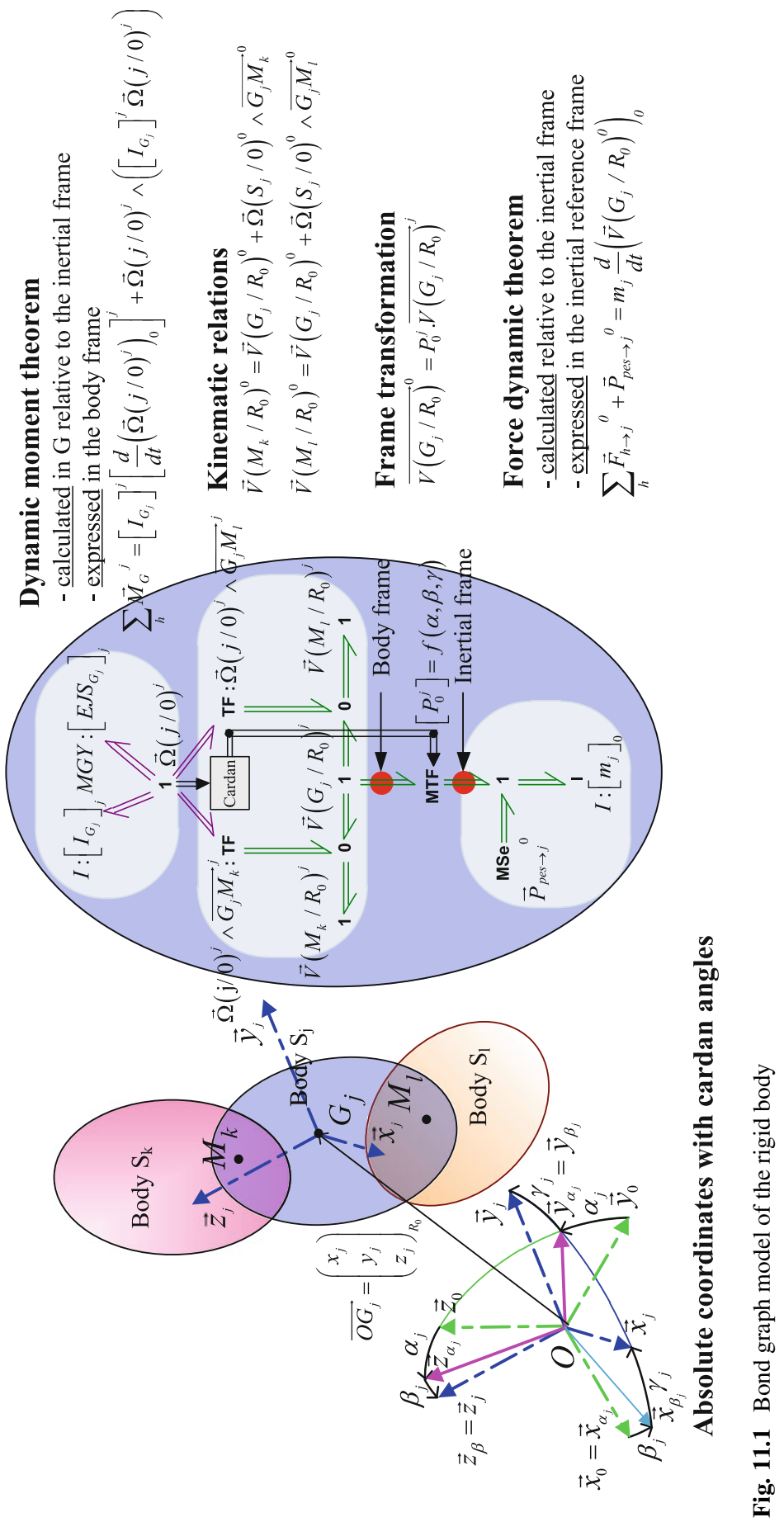




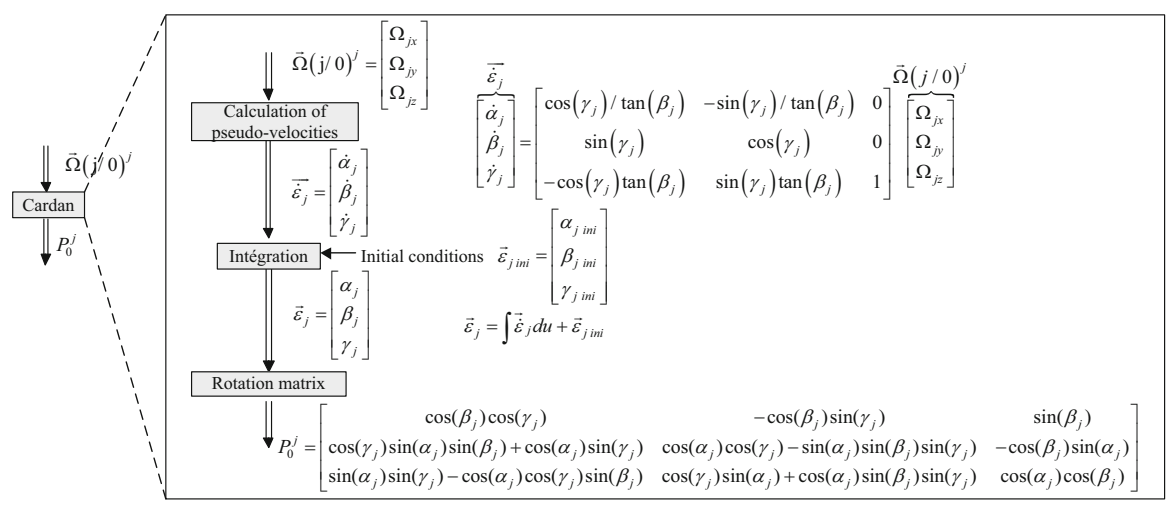

Fig. 11.2 Calculation of the Cardan angles and rotation matrix from the angular velocity

\begin{tabular}{|c|c|c|c|}
\hline $\begin{array}{l}\text { Flow in } \\
\vec{e}_{1}=A \vec{e}_{2}\end{array}$ & $\begin{array}{c}\text { Flow out } \\
\vec{e}_{2}\end{array}$ & $\begin{array}{l}\text { Flow in } \\
\vec{e}_{1}=A \vec{f}_{2}\end{array}$ & $\begin{array}{c}\text { Effort out } \\
\vec{e}_{2}=A^{T} \vec{f}_{1}\end{array}$ \\
\hline $\overrightarrow{f_{1}}$ & $\overrightarrow{f_{2}}=A^{T} \overrightarrow{f_{1}}$ & $\vec{f}_{1}$ & $\vec{f}_{2}$ \\
\hline
\end{tabular}

Fig. 11.3 Causalities imposed with TF and GY elements

The second causality constraint $(\mathrm{C} 2)$ is: the causality of transformers implied in cross products and the causality of gyrators in the bond graph model of the rigid body are intrinsically fixed, the transformers implied in cross products must be flow-in-flow-out causality, and the gyrators must be flow-in causality. Indeed, contrary to scalar bond graphs, the ideal two-ports elements cannot propagate causality in both directions when the moduli are not invertible. In the frame studied (multibody system modeling with bond graphs and the Tiernego and Bos approach), these cases in which moduli (matrices associated to the elements) are not invertible are present for two elements: the transformer (TF) between the rotational and translational domain and the gyristor (GR) since both elements implement cross products. Consequently, transformers and gyrators have the mandatory fixed causality assignment specified above.

The transformers and gyrators with the acceptable causal forms mentioned are given in Fig. 11.3.

These imposed causalities on the transformers lead to some specific precautions when multibody systems with kinematic loops are considered. Figure 11.4 presents the Bond graph model of a rigid body with vector bonds and the imposed causalities mentioned above. Consequently, in the BG of a rigid body, attaching flow sources (Sf) to more than one hinge point or the center mass at the same time is not possible and leads to causality conflicts. This situation typically occurs when the multibody system is composed with kinematic loops. In [12], the author gives the example of an oscillating bar which, with its two joints, is a closed kinematic chain system with one body. 
Fig. 11.4 Bond graph model of rigid body with vector bonds

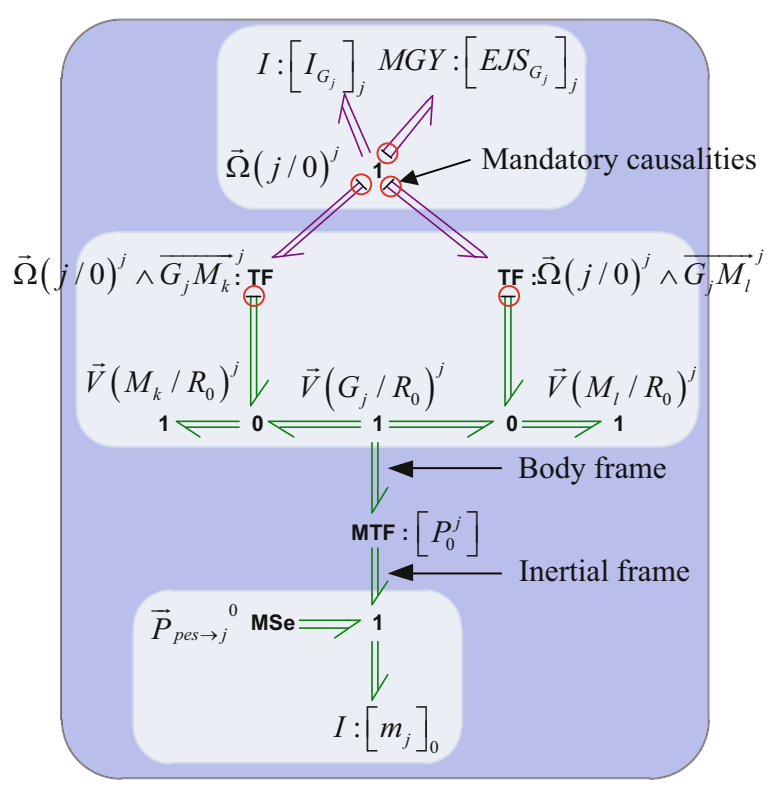

\subsubsection{About DAE Formulation}

In this chapter, in order to keep a modular approach as mentioned in the previous sections, the Tiernego and Bos method has been chosen and then absolute coordinates are selected. It is important to notice that both open chain (OC) and closed kinematic closed (CKC) systems lead to a DAE formulation.

Indeed, due to the kinematic constraints, derivative causality appears on the inertial elements and leads to differential-algebraic equations. In other words, the variables of these inertial elements with derivative causality depend on the variables of the inertial elements with integral causality through algebraic constraints. Consequently, one of the necessary priorities of the simulation method exposed in this paper will be either to handle DAEs using Lagrange multipliers method and specific solvers such BDF or to transform these DAEs it into ODE forms using the parasitic elements method.

\subsubsection{Existing Simulation Methods}

The methods presented in this section come from the references given in Sect. 11.3.1. The added value of this study is to give practical guidelines to modelers so that they can implement the adapted method in 20-sim software. 


\subsubsection{ZCP Method}

\section{General Aspects}

Bond graphs are composed of different types of causal paths. The closed causal paths without integration operations are called zero-order causal paths (ZCP) [11, $13,25,30]$. The mathematical models with ZCPs lead to DAEs. There is a direct link between the nature of the ZCPs in the bond graphs and the index of the DAE's index. The definitions of ZCPs used are those presented in [13] and are the following:

Class 1 ZCPs: The causal path is set between storage ports with integral causality and storage ports with differential causality. The associated topological loops are flat.

Class 2 ZCPs: The causal path is set between elements whose constitutive relations are algebraic (resistors are the most typical case). The topological loops are flat. Class 3 ZCPs: It is a causal cycle whose topological loops are open. The causal path starts and ends in the same port of an element.

Class 4 ZCPs: The Class-4 ZCPs are defined as causal cycles whose topological loops are closed.

Only Class 4 ZCPs lead to DAEs with an index of 2. When the DAE's index is inferior to 2, a Backward Differentiation Formula (BDF) solver (such as the one in 20-sim) can handle these equations. In some cases (scalar bonds or vector bonds with no kinematic loops), the simulation can thus be conducted without the addition of specific elements.

\section{Pros}

The advantages of the ZCP method are as follows. First, this method enables simulations to be conducted directly from the model without requiring the addition of elements (R/C elements or controlled effort sources) at the correction location. The initial physical model is therefore not changed contrary to the $\mathrm{R} / \mathrm{C}$ elements. Secondly, as we will see in Sect. 11.4, this method is faster than the two following methods ( $\mathrm{R} / \mathrm{C}$ elements and controlled effort sources).

\section{Cons}

When multibody systems with kinematic loops are modeled, the bond graph may contain Class-4 ZCPs which lead to a DAE formulation with an index superior to 1 and, then, the BDF solver often encounters difficulties in the numerical computation of the model. The ZCP opening method consists of opening the Class-4 ZCP, thanks to the use of break variables. This technique can be done at an equation level or at a graphical level. The classic technique is to add modulated sources (MSe) at 1junctions of the Class-4 ZCPs so as to open them. Without a systematic approach to 
the detection of the Class-4 ZCPs, this method may be difficult to use when dealing with a case that involves complex multibody systems. This method was thus not used in this chapter in the case of multibody systems with kinematic loops.

\subsubsection{R/C or Parasitic Elements}

\section{General Aspects}

The $\mathrm{R} / \mathrm{C}$ elements (or parasitic elements) method is based on the introduction of: compliances and resistances in the BG models of the joints. This method has been used for a long time. It appeared for the first time with the denomination "Stiffcompliance" approach in [17]. In the literature, other's terminologies can be found for this method: virtual springs [32] or also singular perturbation [35].

In the framework of this chapter (focused on the simulation of multibody systems), this method can have two objectives: eliminating the kinematic constraints and also consequently eliminating the derivative causality or algebraic loops so that an explicit solver may be used. As we have previously mentioned (in Sect. 11.3.1.1), the nature of vector bond graphs imposes some supplementary causality constraints (C1 and C2). For the enforcement of these constraints, the flow sources (Sf) can lead to causal conflicts because of their flow-out causality. The enforcement of constraints with parasitic elements ( $\mathrm{R}$ and $\mathrm{C}$ elements) can circumvent these constraints ( $\mathrm{C} 1$ and $\mathrm{C} 2$ ) instead of flow sources (Sf), thanks to their effort-out causalities. Firstly, it allows the preservation of the same causality assignments for all of the bonds of a multibond (C1 respected). Secondly, it allows the suppression of the causality conflict which may appear because of the imposed causality of the transformers implied in the cross product and gyrators ( $\mathrm{C} 2$ respected).

Moreover, the use of $\mathrm{R} / \mathrm{C}$ elements into joint models decouples the energy store elements associated with the inertia of the rigid bodies. All these energy store elements are then kept with integral causalities.

The values of the compliant elements must be chosen carefully. To our knowledge, two methods for selecting these elements exist: (1) the eigenvalues decoupling between the parasitic frequency and the system frequency, (2) the use of activity metric [24]. These parameters can be chosen so as to model the joint compliances which exist in all mechanical joints. Thus, this point gives to this method a physical significance. The stiffnesses introduced should be high enough so as not to change the dynamic of the system but not too high so as to prevent the numerical difficulties of stiff problems (with high-frequency dynamics). This method leads to a necessary compromise between the accuracy of the results and the simulation time: the stiffer the system is, the more numerical errors are reduced but the more simulation time remains important. However, the increase of the simulation time can be balanced by parallel processing as the mass matrix in a block-diagonal form can be used to 
decouple the system as it is explained in [32]. As T. Rayman recommends, adding a damping element ( $\mathrm{R}$ resistive element) in parallel with the stiff spring ( $\mathrm{C}$ energy store element) enables the dampening of the high eigen frequency associated with the high stiffness. The exact influence of these parameters still remains a topic of research in which the authors are particularly interested in.

\section{Pros}

With the RC elements method, all the derivative causalities are removed. This leads to an explicit ODE model which can be solved with a fixed step solver such as Runge Kutta 4.

Contrary to the Lagrange multipliers methods, some slight imprecisions could be made on the initial conditions. Moreover, overconstrained (also called hyperstatic) systems can be simulated without any difficulties.

\section{Cons}

This method introduces new elements ( $\mathrm{R}$ and $\mathrm{C}$ elements) in the initial bond graph whose parameters and initial conditions associated with the $\mathrm{C}$ store elements need to be specified. The values of the compliant elements must be chosen carefully so as to model the joint compliances which exist in all mechanical joints. The stiffnesses that are used should be high enough so as not to change the dynamics of the system and should be able to sufficiently approximate the constraints. However, too high stiffnesses introduce numerical difficulties of stiff problems (with high-frequency dynamics). Indeed, these kinds of problems force the solvers to take very small integration steps to meet the tolerance criteria and, consequently, the simulation is slowed. This method leads to a necessary compromise between the accuracy of the results and the simulation time: the stiffer the system is, the more numerical errors are reduced but the more the simulation time remains important.

\section{Implementation}

Even if explicit solvers can be used, the authors recommended the use of the implicit Modified Backward Differentiation Formula (MBDF) solver for two reasons. The first reason is that the BDF solver guarantees the numerical stability of the solution. The second reason is that, with the controlled effort sources, only the MBDF solver can be used. Also, using an MBDF solver with the R/C Elements is a good way to facilitate comparison between R/C elements and controlled effort sources methods. 


\subsubsection{The Lagrange Multipliers Method}

\section{General Aspects}

In the "Lagrange-multiplier method," the constraint forces are not modeled by parasitic elements but by controlled effort sources (MSe). The origin of this method comes from [7, 14] and has been implemented in 20-sim in [5, 6, 31]. The same concept was also implemented by [12] with pseudoflow source (PSf) elements.

The general idea of this method is similar to the parasitic elements that is to say: removing the flow sources (Sf) and enforcing the constraint by other elements (here the controlled effort sources MSe)) which don't have flow causality and thus suppress the causality conflict.

The controlled effort sources (MSe) are computed such that the difference between velocities for the constraints is zero. The principle of the method is to apply an effort equivalent to the one that the system would impose on a flow source. It would have a practical operation as a flow source but with effort-out causality instead of a flow-out causality.

The constitutive laws of the controlled effort sources are the following:

- the constitutive law of an effort source,

- the following constraints: effort $=\mathrm{e}$ such as flow $(\mathrm{e})=\mathrm{f}$.

Figure 11.5 may help to understand the implementation of this constraint. This implementation uses a specific function provided by the 20-sim software: constraint() function. At every simulation step, this function induces an iterative procedure to find exactly the force that keeps the velocity offset at zero. This iterative procedure is only supported by the Modified Backward Differentiation Formulation method of the 20-sim software.

Due to the nature of the causality of the controlled effort sources (effort-out causality), all the inertial elements (I elements) receive integral causality. The dependent states are thus not visible in the form of derivative causality. However, a controlled effort source (MSe elements) establishes itself algebraic dependencies with the state variables of the inertia and thus indicates an implicit form of equations. As it is mentioned in [5], the mathematical model obtained (a semi-explicit state space model) can be solved by a BDF solver although the DAE system is index 2 due to the fact that the constraint forces do not appear in the algebraic constraints.
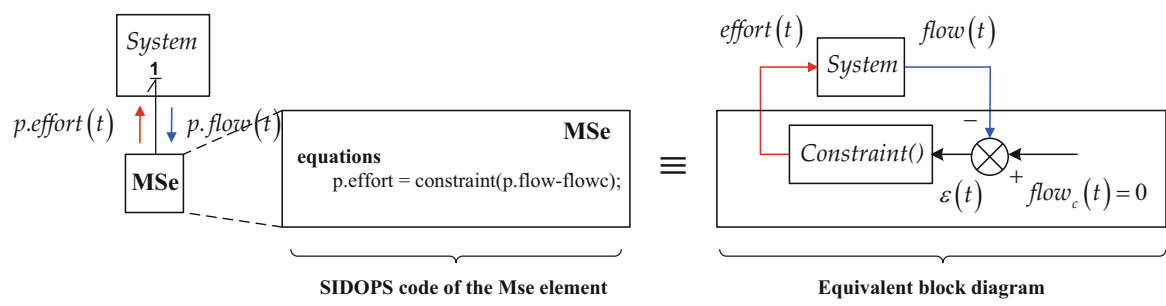

Fig. 11.5 Implementation of the controlled effort source 
Pros

Contrary to the parasitic elements method, the modulated sources (MSe) don't need additional tuning because no additional parameters are added to the system. In other words, the order of the system is not modified because no new states (with associated parameters and initial conditions) are introduced. Moreover, the absence of supplementary parameters makes a bond graph that describes the system ideally in the limit of the numerical tolerance on the constraints equations.

Even if, during the simulation, iterations are required at each time step to satisfy the constraint equations, the computational load is comparable or better in comparison to the parasitic elements method where the differential equations are truly explicit but very stiff.

\section{Cons}

The bond graph obtained with this approach leads to implicit differential equations, so explicit integration algorithms cannot be used. Moreover, during the simulation the constraints can only be met within some numerical tolerances.

Consequently, the implementation of this method requires special care, which is described hereafter.

\section{Implementation}

The Lagrange multipliers method is more difficult to implement than the use of RC elements because the kinematic constraints imposed by the joints may produce computational conflicts. This issue occurs on closed kinematic chains with overconstrained multibody systems. Due to the topology of the system, more than one joint could impose the same constraint on the system, in which case the simulation will no longer be possible. In other ways, more practically, the number of the controlled effort sources must not exceed the degrees of freedom that need to be eliminated. In order to solve this problem, the redundant constraints must be removed.

In addition, there may be the inconsistent initial conditions. Indeed, initial conditions of the system should be consistent with constraints. If a controlled effort source is attached to a 1-junction to keep the velocity at zero and a nonzero initial velocity to the I element connected to the same 1-junction is imposed, then a simulation cannot be conducted. We can notice at this point that 20-sim does not offer an automatic correction of inconsistent initial conditions but, a BG modeler can always code the calculation of consistent initial conditions to handle it automatically. 


\subsubsection{Kinematic Joints Modeling}

The joint models permit the expression of the constraints that are introduced when rigid bodies are connected. As the bond graph model of the rigid body, the joint models have been built in a modular way in the sense that their modeling does not change when the whole model of the system is assembled. The idea of this section is to allow the BG practitioners to be able to use a library of all the common existing kinematic joint models.

\subsubsection{Common Aspects}

The modeling of kinematic joints determines the rotational or translation degree of freedom allowed by the joint.

Flow sources can be used to suppress the joint's degrees of freedom. However, in order to circumvent the causality constraints mentioned before, the joints models are presented with an $\mathrm{L}$ element. The element $\mathrm{L}$ stands either for an $\mathrm{R} / \mathrm{C}$ element or a modulated effort source MSe depending on the choice of the simulation methods ( $\mathrm{R} / \mathrm{C}$ elements methods or the use of Lagrange multipliers).

For the mobilities let free, the choice of modeling assumptions can lead to add elements. If the joints are assumed to be perfect that is to say without any dissipating or energy storage phenomenon, the junction 1 can be let free of elements. If, however, dissipating or spring phenomenon are assumed in the joints, $\mathrm{R}$ or $\mathrm{C}$ elements can be added at these 1 junctions. One can notice that these $\mathrm{R} / \mathrm{C}$ elements shouldn't be confused with the R/C elements modeling parasitic elements and will be called functional elements in Sect. 11.4.

The joint model is built from the following kinematic relationships:

$$
\vec{\Omega}(1 / 0)^{1}=\vec{\Omega}(1 / 2)^{1}+\vec{\Omega}(2 / 0)^{1}
$$

where

$\vec{\Omega}(1 / 0)^{1}$ is the absolute velocity of body 1 expressed in $R_{1}$ frame

$\vec{\Omega}(1 / 2)^{1}$ is the relative angular velocity of body 1 with regard to body 2 expressed in $R_{1}$ frame

$\vec{\Omega}(2 / 0)^{1}$ is the absolute velocity of body 2 expressed in $R_{1}$ frame and

$$
\begin{aligned}
\vec{V}\left(O_{2} \in 2 / 0\right)^{1} & =\vec{V}\left(O_{2} \in 2 / 1\right)^{1}+\vec{V}\left(O_{2} \in 1 / 0\right)^{1} \\
& =\vec{V}\left(O_{2} \in 2 / 1\right)^{1}+\left(\vec{V}\left(O_{1} \in 1 / 0\right)^{1}+\vec{\Omega}(1 / 0)^{1} \wedge{\overrightarrow{O_{1} O_{2}}}^{1}\right)
\end{aligned}
$$

which can be also written as 
Fig. 11.6 General kinematic joint model

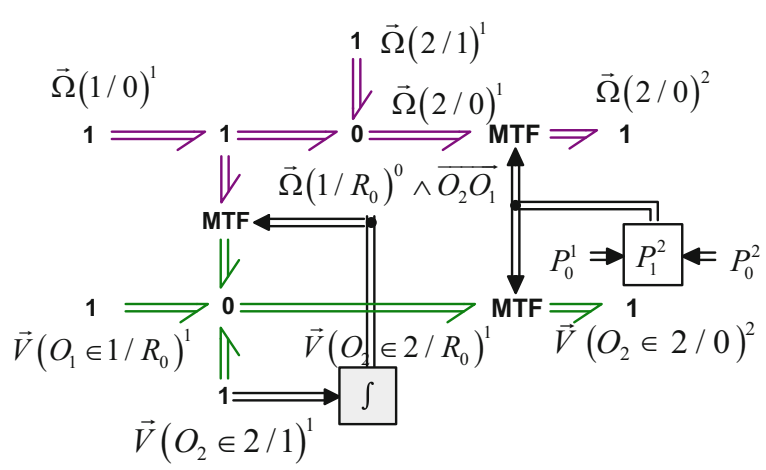

$$
\vec{V}\left(O_{2} / 0\right)^{1}=\vec{V}\left(O_{2} \in 2 / 1\right)^{1}+\left(\vec{V}\left(O_{1} / 0\right)^{1}+\vec{\Omega}(1 / 0)^{1} \wedge{\overrightarrow{O_{1} O_{2}}}^{1}\right)
$$

where

$\vec{V}\left(O_{2} / 0\right)^{1}$ is the absolute velocity of point $O_{2}$

$\vec{V}\left(O_{2} / 0\right)^{1}$ is the absolute velocity of point $O_{1}$

$\vec{V}\left(O_{2} \in 2 / 1\right)^{1}$ is the relative velocity of point $O_{2}$ with regard to the frame $R_{1}$

$\vec{\Omega}(1 / 0)^{1} \wedge{\overrightarrow{O_{1} O_{2}}}^{1}$ is the velocity component due to the rotation of frame $R_{1}$ with regard to the inertial reference frame

The general kinematic joint model is then detailed in Fig. 11.6.

In the general kinematic joint model, MTF elements modulated by coordinate transformation matrix are used so as to express the kinematic quantities in the appropriate frame associated with the body where they are connected to.

\subsubsection{Joint Models}

Based on the general kinematic joint model, the models of the common kinematics joints are given in Figs. 11.7, 11.8, and 11.9.

\subsection{Presentation of the Vibration Absorber System}

\subsubsection{Context}

The rotor of a helicopter can generate various vibration phenomena. Let us consider:

- forced vibrations, 


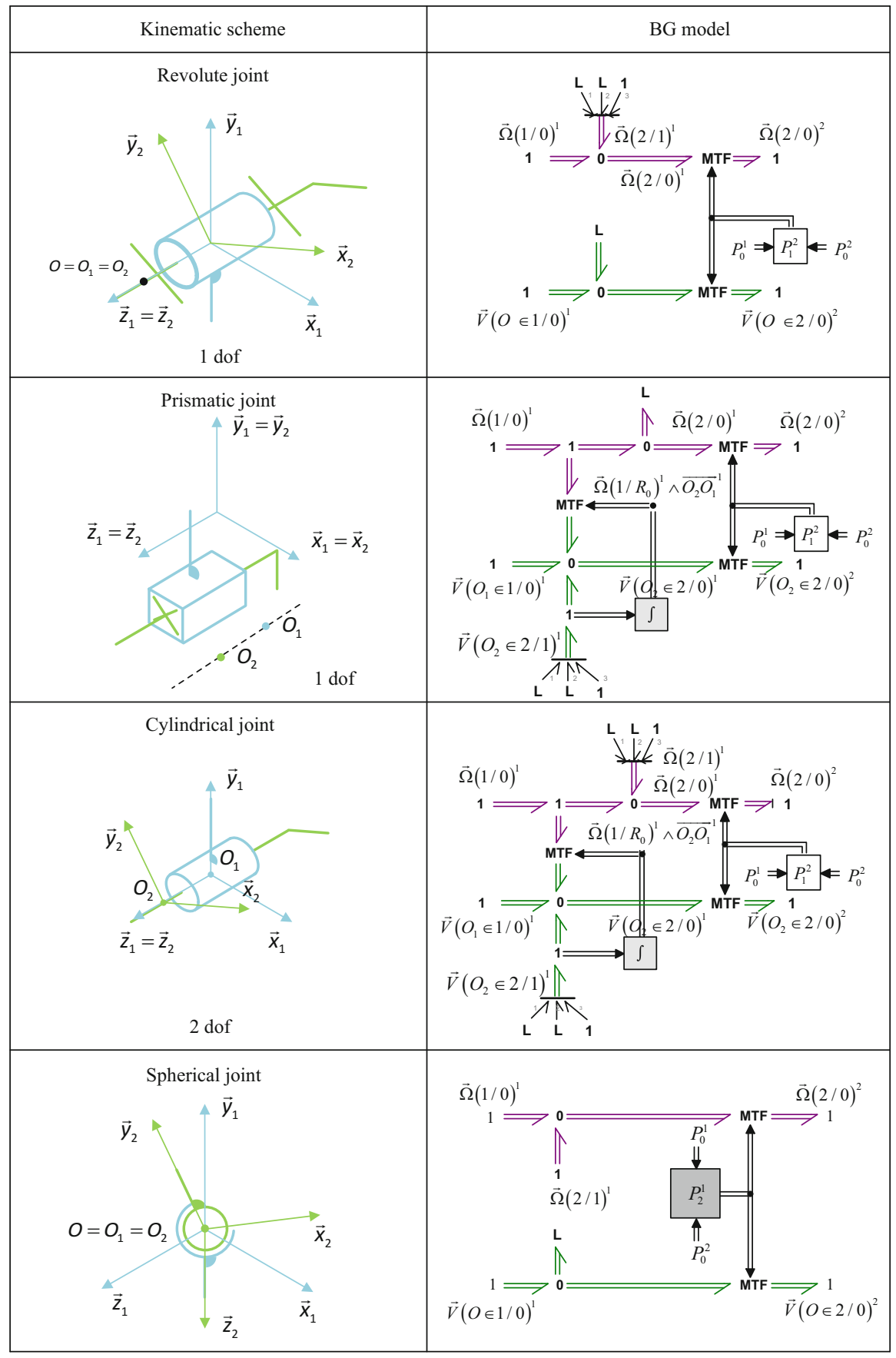

Fig. 11.7 Kinematic joint models-part 1 


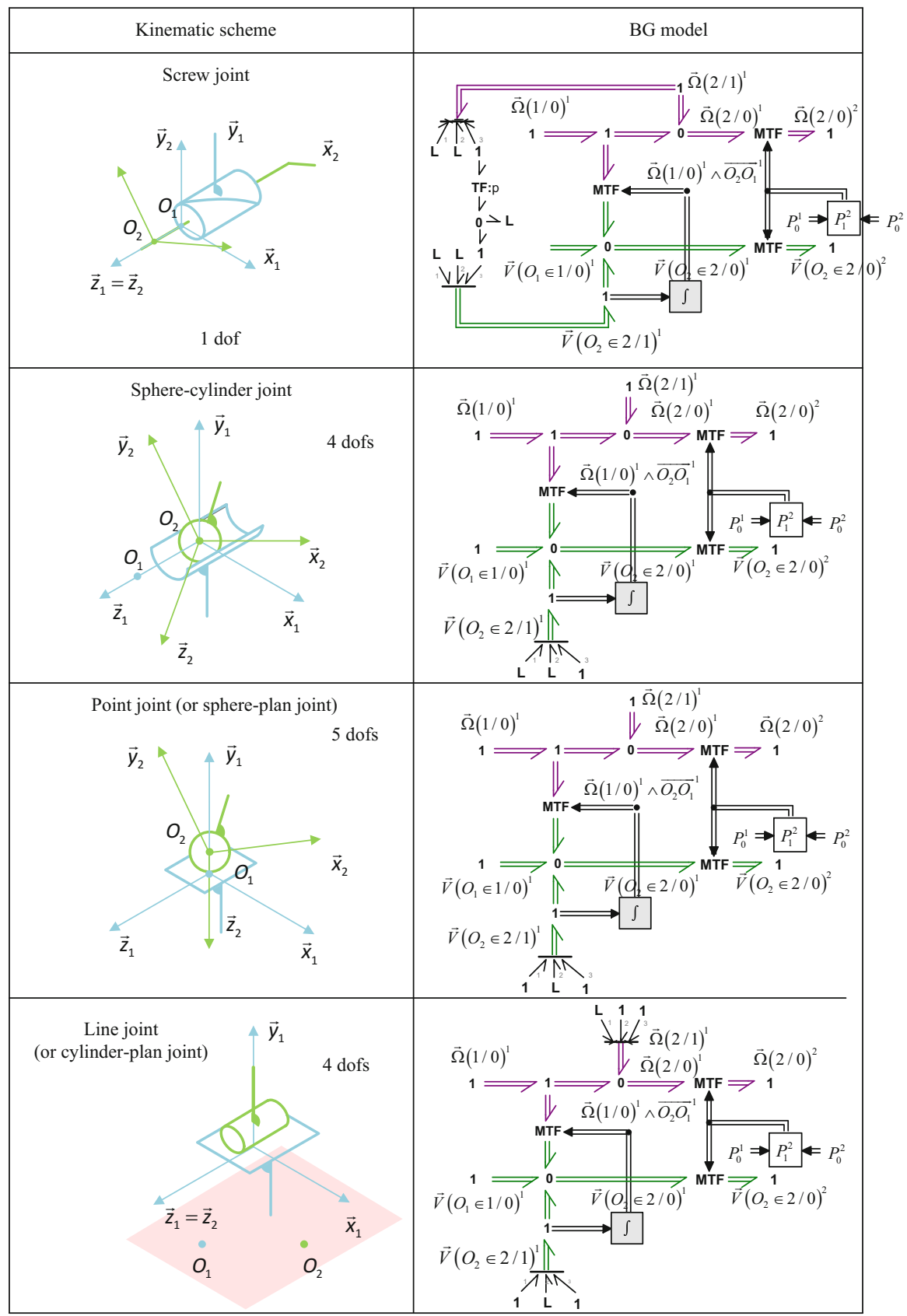

Fig. 11.8 Kinematic joint models-part 2 


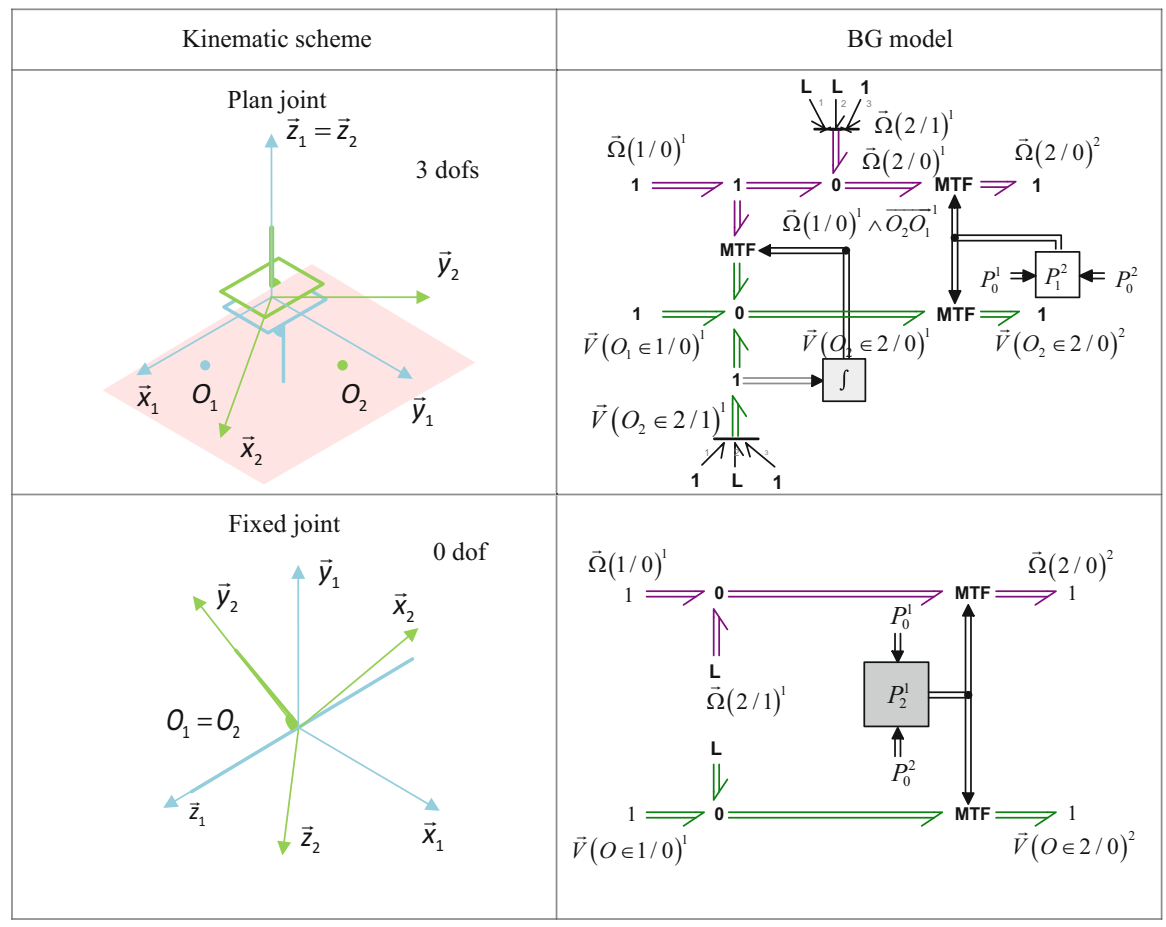

Fig. 11.9 Kinematic joint models_-part 3

- resonances "ground and air,"

- dynamic problems of the power chain.

Blades undergo periodic and alternating inertia and aerodynamic forces whose fundamental frequency is the rotation frequency of the rotor as explained in [18]. These efforts on the blades cause forces and torques on the hub which then becomes a mechanical excitation of the fuselage. It is always shown in [18] that these forces and moments transmitted from the rotor to the fuselage have frequencies whose are frequency harmonics $(\mathrm{kb} \Omega)$ with $\mathrm{b}$ the number of blades of the main rotor and $\Omega$ the rotation speed of the main rotor. Moreover, most of the time, the first harmonic $\mathrm{b} \Omega$ is preponderant.

Therefore, its behavior depends on its dynamic characteristics and the filtering systems placed between the rotor and the fuselage (as shown in Fig. 11.10). In this sequel, we will focus on a vibration absorber system: the SARIB system (called Suspension Antivibratoire à Résonateur Intégré dans les Barres in French). 


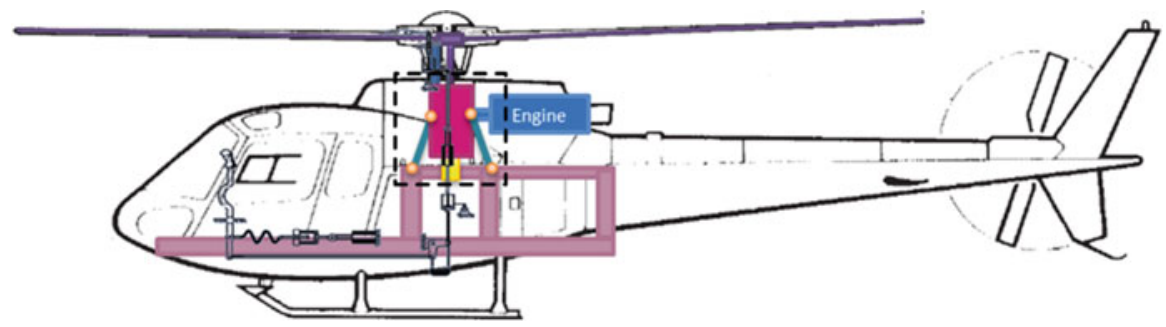

Fig. 11.10 Helicopter suspension between the MGB and the aircraft structure

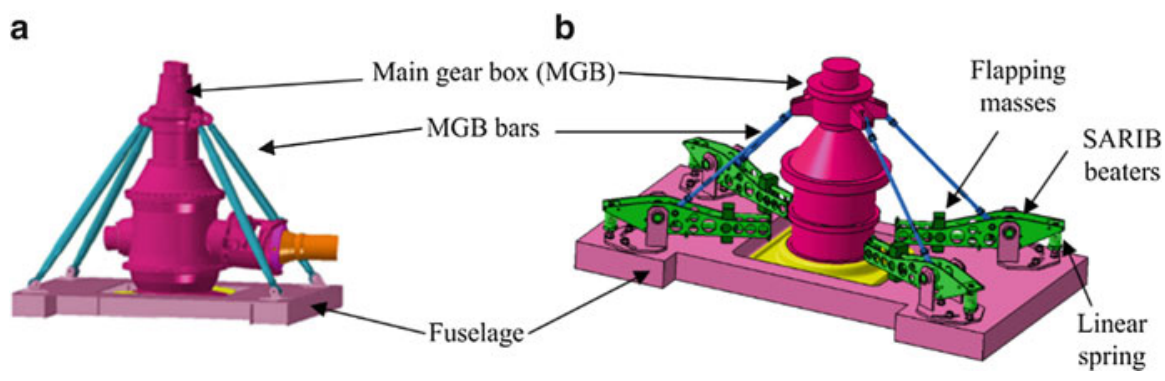

Fig. 11.11 (a) Flexible classical MGB-fuselage suspension, (b) MGB-fuselage suspension with SARIB system

\subsubsection{Interest and Functions}

The MGB-fuselage suspension must ensure several important functions. Firstly, the joint allows the transmission of the static force necessary to the sustentation of the helicopter with a limited static displacement. Moreover, the suspension helps to reduce the mechanical vibrations transmitted to the fuselage according to the force and displacement aspects. In this paper, we will focus on this latter function.

\subsubsection{Operating Principle}

The classical MGB-fuselage suspension is composed of four MGB bars and a main membrane as shown in Fig. 11.11a. The MGB bars can rigidly suspend the fuselage without flexibility to the rotor and thus transmit the lift from the rotor to the structure.

The membrane is a flexible suspension with the following observed behavior:

- a low stiffness for angular movements around the roll and pitch axes and the linear vertical pumping displacement,

- a very high stiffness for linear movements perpendicular to the vertical direction and for the yaw movement. 
Thus, the membrane allows the angular movement of the MGB around the pitch and roll axes. The flexibility of the membrane around these axes allows a strong filtration of the dynamic moments around these axes. In addition, the membrane transmits the main rotor torque, thanks to its very high stiffness around the yaw axis. In conclusion, the conventional suspensions allow filtering pitch and roll dynamic moments without filtering the pumping dynamic efforts.

The suspension equipped with SARIB system is a tuned-mass vibration absorber. Its purpose is to render the filtering of these pumping dynamic efforts possible. On the SARIB solution, beaters with flapping masses are added between the MGB bars and the fuselage (Fig. 11.11b). They permit a vertical degree of freedom to the MGB with regard to the fuselage. The principle of the SARIB system is to tune, for an accurate defined frequency, these flapping masses on each SARIB bar so as to create inertial forces on the fuselage opposite to the force of the MGB bars on the beaters. Moreover, the specificity of the SARIB system among the class of tunedmass absorbers comes from the fact that a rotating SARIB bar works as a mechanical gear ratio that makes the resonating flapping mass appear larger. More details on this operating principle are given in [15] through the equilibrium of an SARIB bar. In other words, there is an anti-resonance phenomenon on the transmissibility function between the excitation applied to the MGB. Hence, the forces transmitted to the fuselage can be strongly reduced at this anti-resonance frequency.

\subsubsection{Use of the Experimental Setup}

To analyze the simulation results, an experimental setup on a small scale has been used. The geometry and mass properties correspond to a light helicopter with a scale of approximately $1 / 2$. The model proposed in this chapter has been simulated with the numerical data of this experimental setup detailed in [8].

The experimental setup has to represent the flight behavior of the helicopter in different operating phases: hover, up/down, or turn. In these operating phases, the MGB is subjected to pumping, pitch, and roll excitements. On the experimental setup, the rotor action, provided by the aerodynamic forces and the blades stiffness, applied to the MGB will be approximated by a sinusoidal excitation at the frequency $\mathrm{b} \Omega$ obtained with a vibration equipment. The experimental setup was performed by maintaining the architecture of a real device (Fig. 11.12).

The correspondence between the different mechanical elements of a helicopter and the experimental setup will be detailed hereafter.

To reproduce the effects of the rotor lift, the set \{BTP-link-fuselage\} has been suspended from the fixed frame (Fig. 11.12). This joint has to lift up the entire weight suspended by analogy to the static force of lift, it was performed by the use of pneumatic components. They were designed so that the natural frequency induced by the overall stiffness is below $2 \mathrm{~Hz}$.

The fuselage was replaced by a rigid mass called fuselage mass (Fig. 11.12). 
Fig. 11.12 Experimental setup of the semi-active suspension

Fig. 11.13 Position of the fixtures to the fuselage
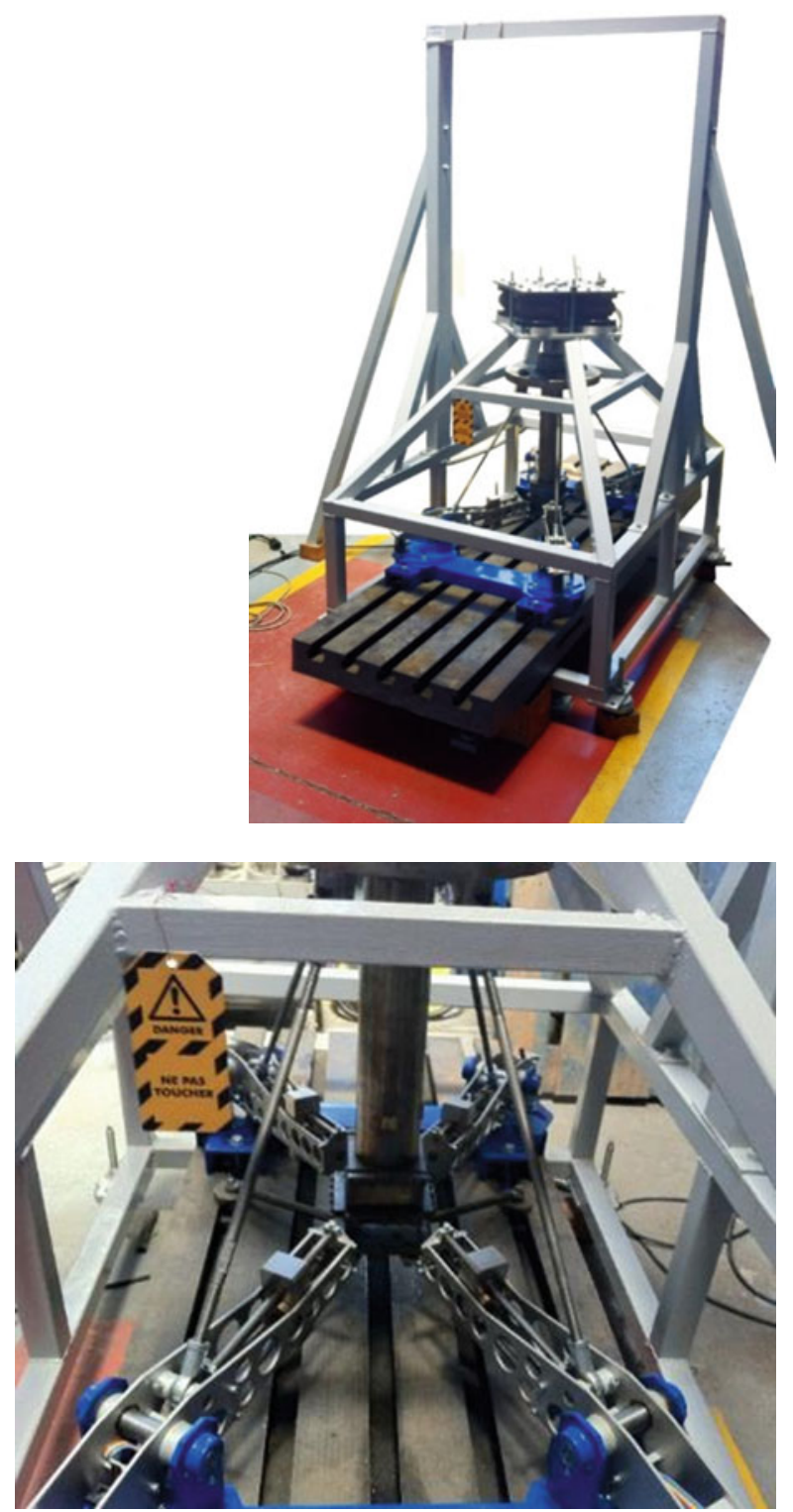

A single body has replaced the set \{rotor shaft-MGB\} and the rotation has been deleted. This single body is composed of a shaft equipped with a recessed tray (Fig. 11.13).

The overall joint between the fuselage mass and the system equivalent to the set \{rotor shaft-MGB\} has four bars, four SARIB beaters, and a system representing the usually existing membrane on the helicopter. 
Fig. 11.14 Revolute joint between an SARIB beater and the fuselage

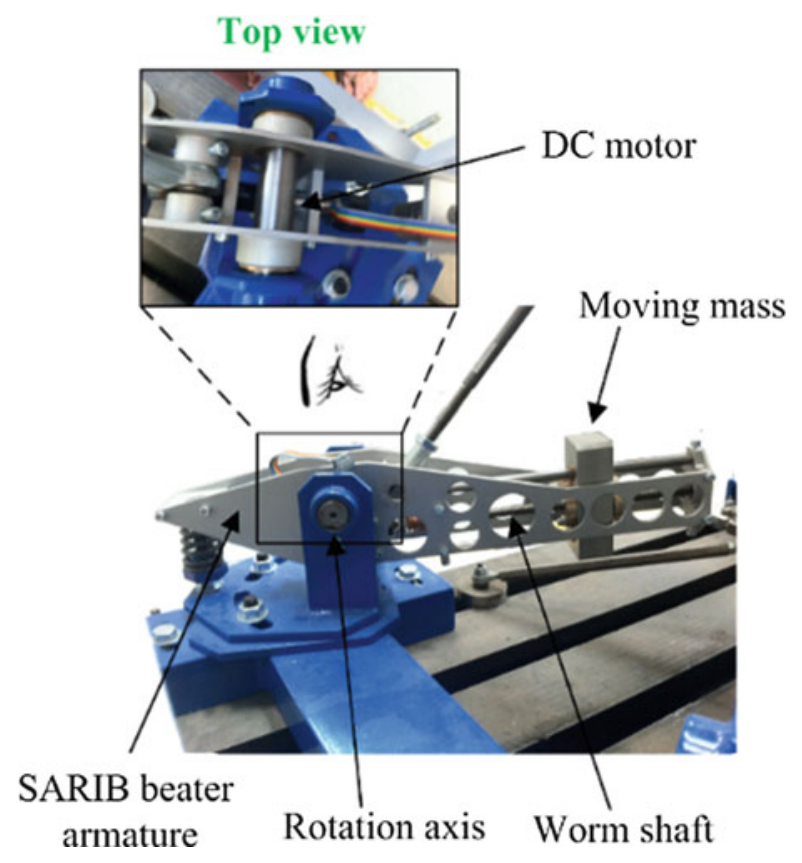

The MGB bars consist of threaded rods that have a spherical joint without backlash at each end.

Each SARIB beater is linked by a revolute joint to the fuselage, which are themselves clamped to the fuselage mass as shown in Fig. 11.13. Each SARIB beater is fitted with a moving mass (Fig. 11.14) connected with a prismatic joint along the SARIB beater. It should be noted that, for the passive suspension presented in this chapter, the moving masses are fixed on the SARIB beaters.

\subsection{Modeling of the Vibration Absorber Suspension}

The bond graph modeling steps described above will now be applied to model the MGB-fuselage suspension equipped with the SARIB device. First, the mechanical model of the MGB-fuselage suspension and the associated assumptions will be presented. Secondly, the construction of the bond graph model of the MGB-fuselage suspension will be detailed. 


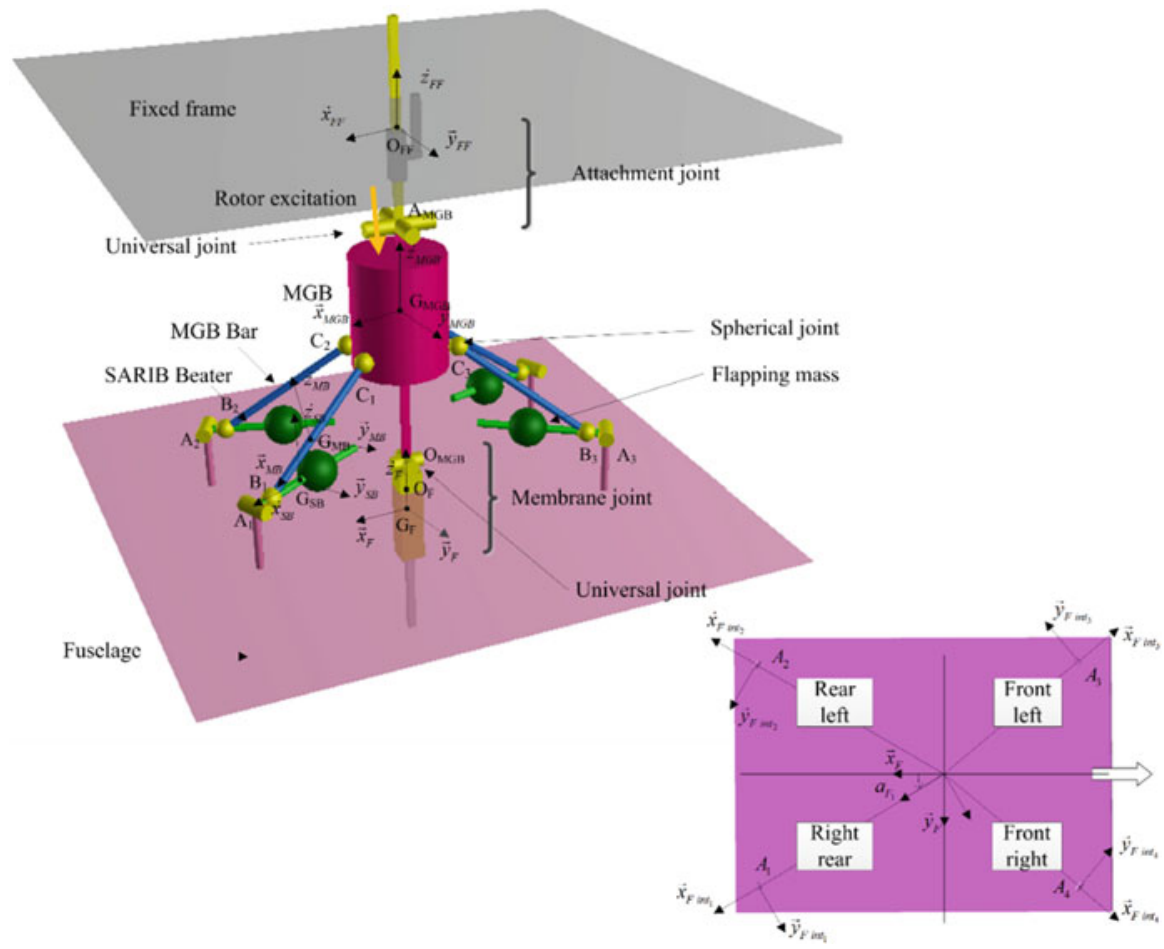

Fig. 11.15 Kinematic scheme of the 3D joint between the main gearbox and the fuselage

\subsubsection{Modeling Assumptions of the Vibration Absorber Suspension}

The 3D vibration absorber suspension is a set of 14 rigid bodies (the MGB, four MGB bars, four SARIB beaters, four intermediate bodies, and the fuselage) and 18 kinematic joints (four revolute joints (R), eight spherical joints (S), and two prismatic joints $(\mathrm{P})$ ). The kinematic scheme of the 3D MGB-fuselage suspension is shown in Fig. 11.15.

For the sake of clarity, the different kinematic joints are also described in the joints graph (Fig. 11.16).

The MGB is suspended by a joint called attachment to the fixed frame. The role of this joint is to support the weight of the system and consequently let the fuselage with six degrees of freedom in the air. This joint consists of a universal joint and a prismatic joint. The weight of the system (mainly the weight of the MGB and the fuselage) is taken up by a tension spring at the prismatic joint of this attach. The spring is dimensioned such that the natural frequency of the mass/spring system is small enough and distant from the excitation frequency applied to the MGB. 


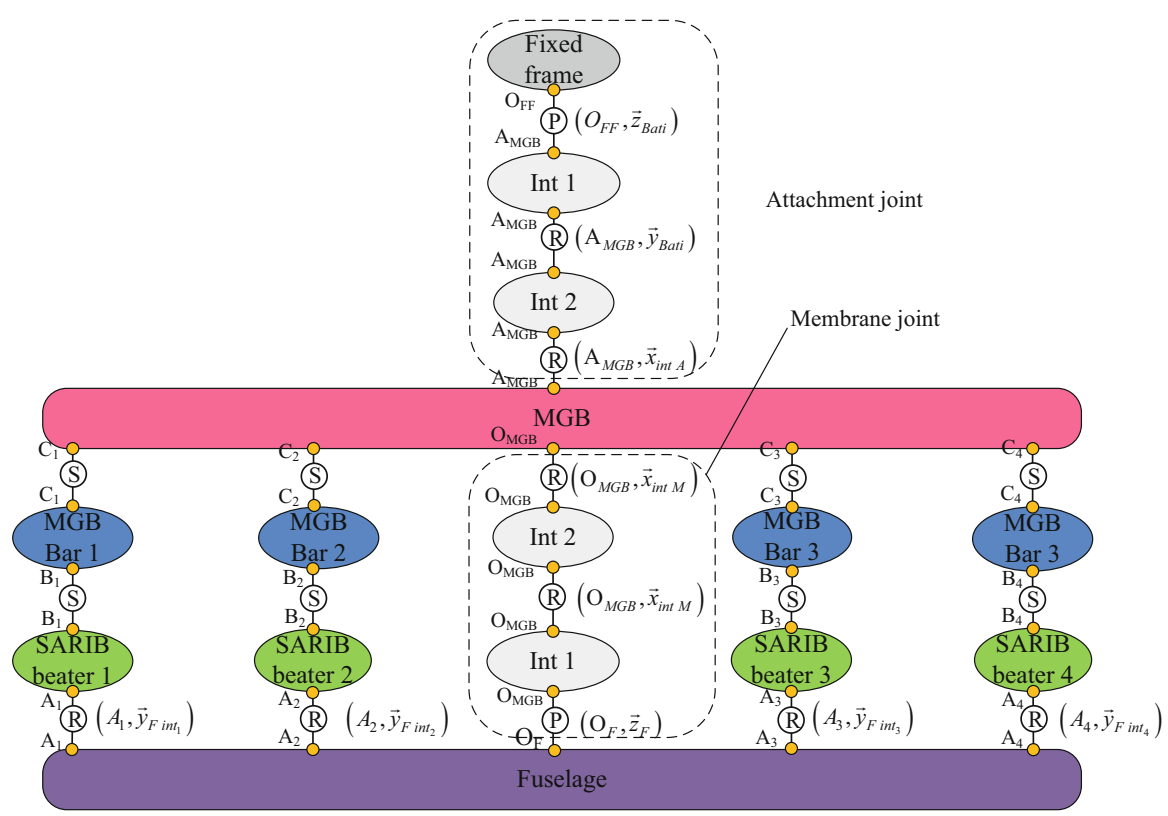

Fig. 11.16 Joints graph of the 3D vibration absorber suspension

The structure of the suspension consists of four identical legs and a central membrane. Each leg consists of SARIB beaters and an MGB bar connected by a spherical joint. The upper ends of these legs are connected to the MGB with spherical joints and the lower ends of these legs are connected to the fuselage through revolute joints. The revolute joints between SARIB beaters and the fuselage have a torsion spring dimensioned such that the anti-resonance frequency in the efforts transmitted to the fuselage is positioned on the rotor excitation frequency while taking up enough effort to lift the helicopter with a small displacement between the MGB and fuselage.

Given the physical phenomena observed, the main membrane between the MGB and the fuselage is modeled by two revolute joints with orthogonal axes (also called universal joint) and a prismatic joint in serial. The flexibilities of the membrane in pumping, roll, and pitch are modeled by the degrees of freedom of these joints combined with stiffness. A torsion spring with low stiffness at each revolute joint of the universal joint and a linear spring also with low stiffness along the $z$-axis will model the filtering carried out by the membrane. The rigidities of the membrane are modeled by rigid joints or, in other words, by a lack of degree of freedom around or along the movements considered.

These bodies are assumed to be rigid. The intermediate parts (called Int 1 and Int2) are supposed with negligible masses. As previously mentioned, absolute coordinates have been chosen for modularity reasons. Some local moving reference frames are attached to these bodies: 
$R_{\mathrm{FF}}=\left(O_{\mathrm{FF}}, \vec{x}_{\mathrm{FF}}, \vec{y}_{\mathrm{FF}}, \vec{z}_{\mathrm{FF}}\right)$ attached to the fixed frame,

$R_{\mathrm{MGB}}=\left(G_{\mathrm{MGB}}, \vec{x}_{\mathrm{MGB}}, \vec{y}_{\mathrm{MGB}}, \vec{z}_{\mathrm{MGB}}\right)$ attached to the MGB,

$R_{\mathrm{SB}_{i}}=\left(G_{\mathrm{SB}_{i}}, \vec{x}_{\mathrm{SB}_{i}}, \vec{y}_{\mathrm{SB}_{i}}, \vec{z}_{\mathrm{SB}_{i}}\right)$ attached to the SARIB Beaters,

$R_{\mathrm{MB}_{i}}=\left(G_{\mathrm{MB}_{i}}, \vec{x}_{\mathrm{MB}_{i}}, \vec{y}_{\mathrm{MB}_{i}}, \vec{z}_{\mathrm{MB}_{i}}\right)$ attached to the MGB Bar,

$R_{F}=\left(G_{F}, \vec{x}_{F}, \vec{y}_{F}, \vec{z}_{F}\right)$ attached to the fuselage and $R_{F}$ int $_{i}=\left(A_{i}, \vec{x}_{F}\right.$ int $_{i}$,

$\vec{y}_{F}$ int $_{i}, \vec{z}_{F}$ int $\left._{i}\right)$ also attached to fuselage so as to facilitate the definition of the axis of the revolute joints.

\subsubsection{Modeling Assumptions of the Suspension Environment}

As previously mentioned in Sect. 11.3, the action of the rotor on the MGB in the experimental setup is approximated by a sinusoidal excitation with a frequency $b \Omega$.

The external mechanical actions applied to the MGB are the forces applied by the vibrating shakers. The description of the experimental setup shall be specified in Sect. 11.5.2 of this paper. These excitations will be applied along or around the joints of the "attach" joint between the fixed frame and the MGB. They allow the MGB to be subjected to pumping, pitch, and roll excitations. These mechanical actions will only consist of a dynamic component to analyze the vibration behavior of the suspension.

For the pumping excitation, the wrench applied by the actuator at the mobility of the prismatic joint is

$$
\begin{aligned}
& \tau \text { (actuator along vertical axis } \rightarrow \text { prismatic joint) } \\
& =o_{\mathrm{FF}}\left\{\begin{array}{c}
f(t) \vec{z}_{\mathrm{FF}} \\
\overrightarrow{0}
\end{array}\right\} \text { avec } f(t)=F \times g(t)
\end{aligned}
$$

For roll excitation, the wrench applied by the actuator at the mobility of the revolute joint around roll axis is

$\tau$ (Actuator around roll axis $\rightarrow$ roll revolute joint)

$$
={ }_{A_{\mathrm{MGB}}}\left\{\begin{array}{c}
\overrightarrow{0} \\
m_{r}(t) \vec{y}_{\mathrm{FF}}
\end{array}\right\} \quad \text { avec } \quad m_{r}(t)=M \times g(t)
$$

For pitch excitation, the wrench applied by the actuator at the mobility of the revolute joint around pitch axis is

$$
\begin{aligned}
& \tau \text { (actuator around pitch axis } \rightarrow \text { pitch revolute joint) } \\
& =A_{\mathrm{MGB}}\left\{\begin{array}{c}
\overrightarrow{0} \\
m_{p}(t) \vec{x}_{\text {int }} A
\end{array}\right\} \text { avec } m_{p}(t)=M \times g(t)
\end{aligned}
$$


Different types of excitation are used by modulating the amplitude of each excitation by a dimensionless function $\mathrm{g}(\mathrm{t})$ : constant, sinus type, or swept sine. The constant excitation permits to ensure that the equilibrium position of the system under its own weight is physically acceptable. The sine excitation permits to get more accurate frequencies of the anti-resonance frequencies. The swept sine excitation is used to analyze the frequency behavior of the system including resonances and anti-resonances frequencies.

For the swept sine excitation, a modulation function was constructed, as shown in four parts:

- within $\left[0, t_{1}\right]$, a progressive increase to a sinusoidal excitation at a frequency of $f_{\text {start }}$ de $5 \mathrm{~Hz}$ is carried out with a linear amplitude variation,

$$
\left\{\begin{array}{c}
\forall t \in\left[0, \frac{t_{1}}{2}\right], \quad g(t)=\left(4 \frac{t^{2}}{t_{1}^{2} 2}\right) \sin \left(\omega_{\text {start }} t\right) \\
\forall t \in\left[\frac{t_{1}}{2}, t_{1}\right], \quad g(t)=\left(-\frac{2}{t_{1}^{2}}\left(t-\frac{t_{1}}{2}\right)^{2}+\frac{2}{t_{1}}\left(t-\frac{t_{1}}{2}\right)+\frac{1}{2}\right) \sin \left(\omega_{\text {start }} t\right)
\end{array}\right.
$$

- within $\left[t_{1}, t_{2}\right]$, the sine excited mode at $f_{\text {start }}$ is preserved the necessary time so that the transient mode disappears and there is only permanent regime,

$$
\forall t \in\left[t_{1}, t_{2}\right], \quad g(t)=\sin \left(\omega_{\text {start }} t\right)
$$

- within $\left[t_{2}, t_{3}\right]$, a linear swept sine is used with a sweep frequency from $f_{\text {start }}(5 \mathrm{~Hz})$ to $f_{\text {end }}(25 \mathrm{~Hz})$. The expression of the chirp signal is given in [19] and recalled below :

$$
\forall t \in\left[t_{2}, t_{3}\right], g(t)=\sin \left(\left(\omega_{\text {start }}+\frac{\Delta \omega}{2 \Delta T}\left(t-t_{2}\right)\right) \times t\right)
$$

With $\Delta \omega=\omega_{\text {end }}-\omega_{\text {start }}$ and $\Delta T=t_{3}-t_{2}$

- within $\left[t_{3},+\infty\right]$, the sine excited mode at $f_{\text {end }}=\frac{\omega_{\text {end }}}{2 \pi}(25 \mathrm{~Hz})$ is kept.

$$
\forall t \in\left[t_{3},+\infty\right], \quad g(t)=\sin \left(\omega_{\text {end }} t\right)
$$

For the conducted simulations, the time intervals were defined with the numerical following values: $t_{1}=3 \mathrm{~s}, t_{2}=5 \mathrm{~s}$, and $t_{3}=25 \mathrm{~s}$. The chirp signal is built in a such manner that from $t_{2}$ to $t_{3}$ the excitation at the i s corresponds to an excitation at the i Hz (Fig. 11.17). 


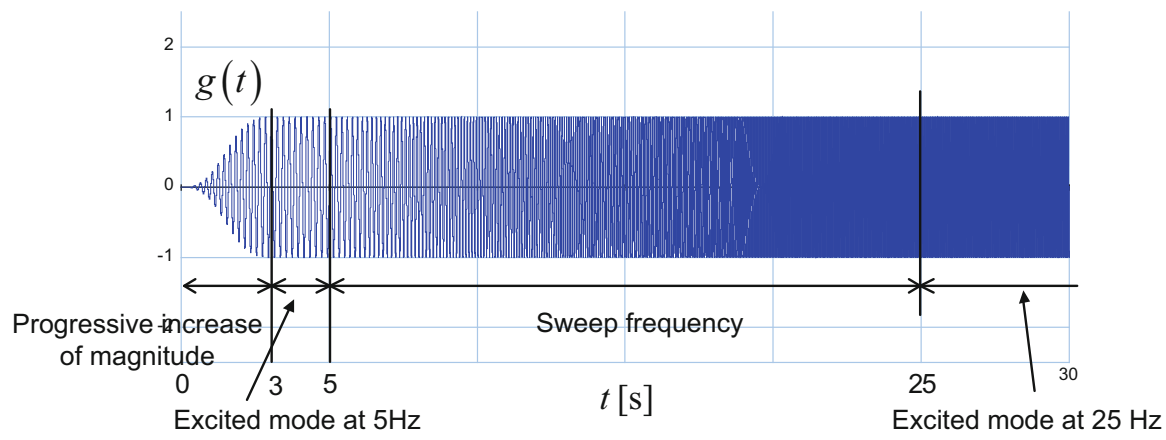

Fig. 11.17 Modulation function of the excitation $g(t)$

\subsubsection{The Simulation Approach Chosen: The R/C or Parasitic Elements Methods}

The bond graph simulation with the $\mathrm{R} / \mathrm{C}$ or parasitic elements method is quite easy to implement compared to conventional techniques since there are no kinematic constraints to deal with. The $\mathrm{R} / \mathrm{C}$ or parasitic elements method allows to tackle derivative causalities which can appear on some inertial elements and causalities constraints due to vector bonds. Moreover, this method, from our point of view, keeps a physical insight and permits the modeling with a modular approach.

\subsubsection{Construction of the Bond Graph Model of the MGB-Fuselage Joint}

\subsubsection{Components Modeling}

The components modeling (rigid bodies and kinematic joints) of the system will now be presented.

\section{Rigid Bodies}

\section{The $M G B$}

The MBG representation of the construction is given in Fig. 11.18. The bond graph representation shows as many branches in the structure junctions as kinematic joints for the determination of the velocities of corresponding points. From the linear velocity of the center of mass $G_{\mathrm{MGB}}$ and the instant rotation velocity $\vec{\Omega}$ (MGB/Fixed frame), the linear velocity of the point $O_{\mathrm{MGB}}$, the linear velocity of 


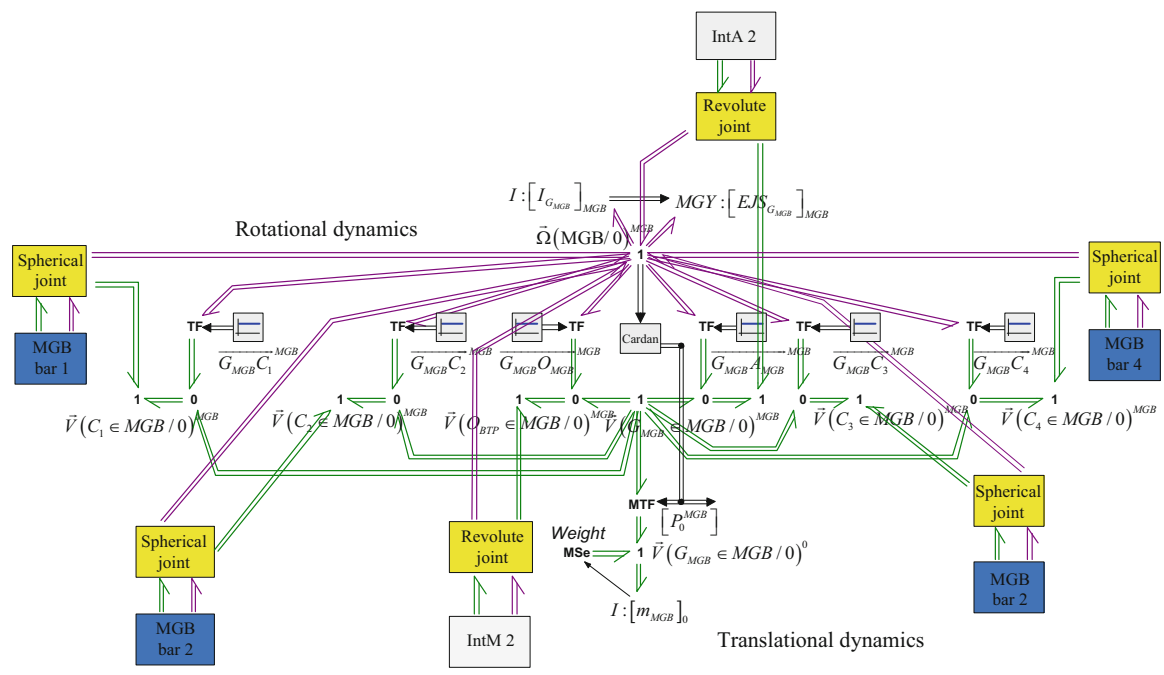

Fig. 11.18 Bond graph modeling of the MGB with its environment

$A_{\mathrm{MGB}}$ point of the MGB, and the linear velocities $C i$ point at the ball joints between BTP and BTP bars are calculated.

\section{The Fuselage}

With a similar approach, the bond graph modeling of the fuselage is given in Fig. 11.19.

\section{The SARIB Beaters}

The bond graph model of an SARIB beater $i$ kinematically linked to the fuselage and the MGB bar $i$ is given in Fig. 11.20.

\section{The MGB Bars}

The bond graph model of an MGB bar $i$ kinematically linked to the MGB and an SARIB bar $i$ is given in Fig. 11.21.

\section{Kinematic Joints}

The joint modeling will now be detailed. The singular perturbation method is here implemented by the addition of parasitic elements which were presented in the previous section. 


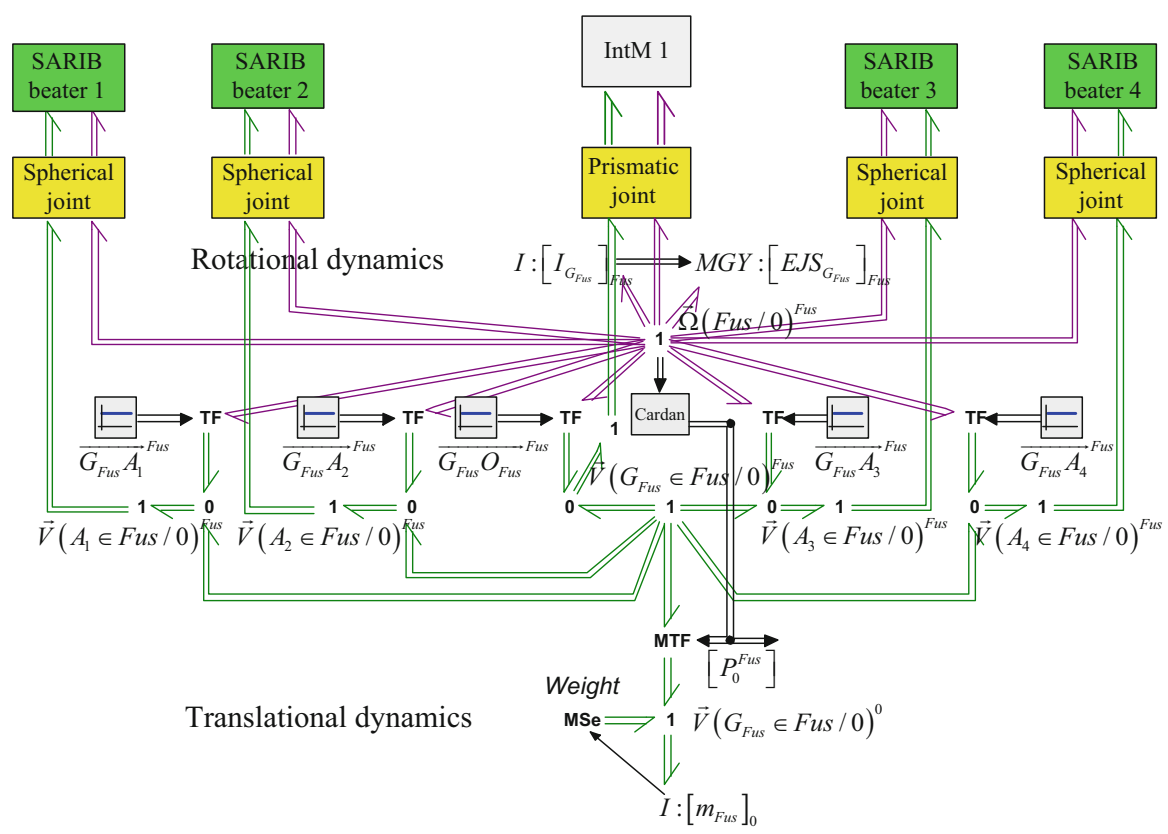

Fig. 11.19 Bond graph model of the fuselage with its environment

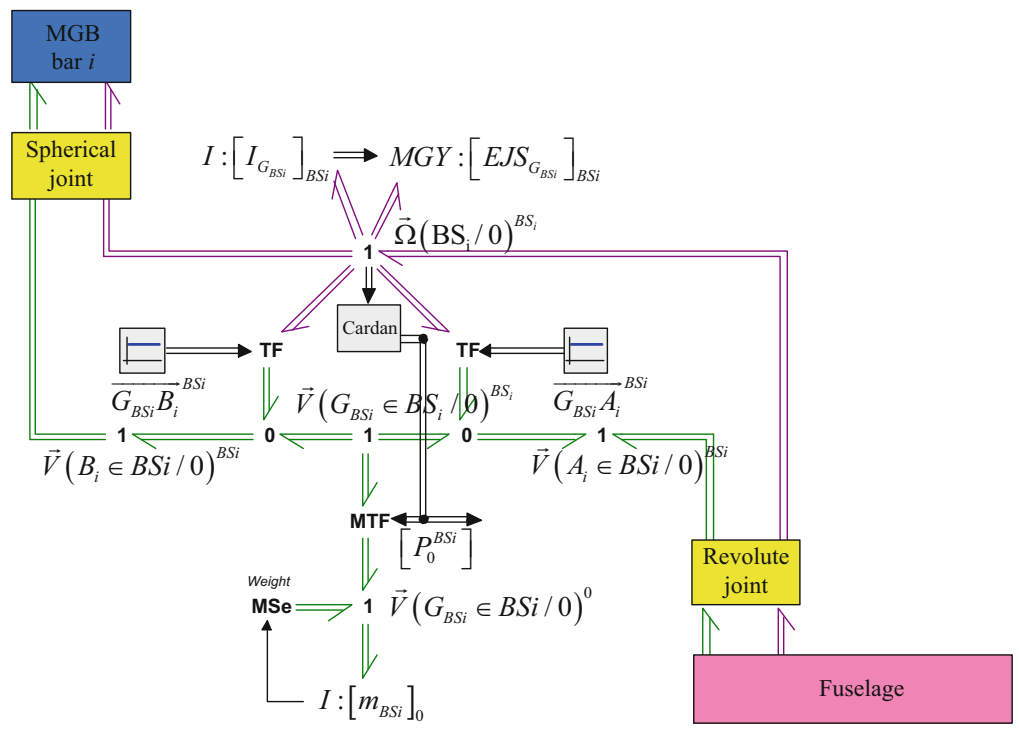

Fig. 11.20 Bond graph model of an SARIB beater $i$ kinematically linked to its environment 


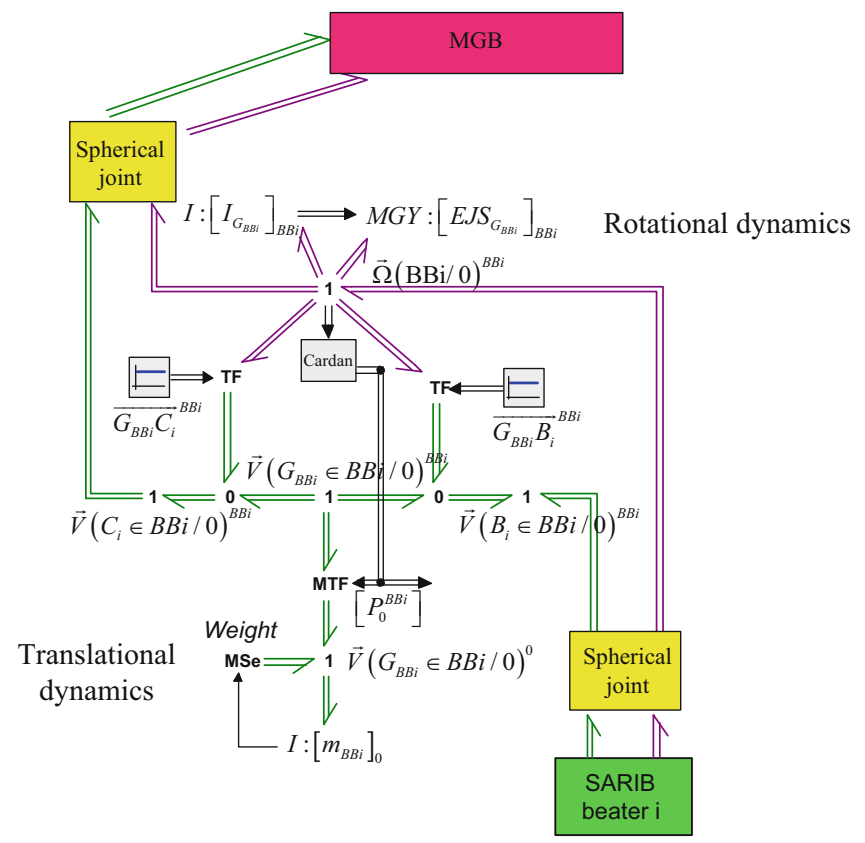

Fig. 11.21 Bond graph model of MGB bar $i$ kinematically linked to its environment

\section{The Revolute Joints}

Eight revolute joints are present in the system: two for the universal joint in the attach joint between the fixed frame and the MGB, two for the universal joint in the membrane between the MGB and the fuselage, and four between each SARIB beaters and the fuselage. The bond graph modeling between the SARIB beaters and the fuselage is given in Fig. 11.22. The bond graph models of the other revolute joints are designed in a similar manner.

The upper part of this joint's model corresponds to the angular velocities. The lower part of this joint's model corresponds to the translational velocities. The R/C elements in this model are functionally different. On the one hand, the $\mathrm{R} / \mathrm{C}$ elements corresponding to the stiffness and damping around the y axis (revolute joint's axis) model the behavior of the torsional spring. On the other hand, the R/C elements with high stiffness and some damping around the other axes model the virtual springs since there are no physical springs in these directions. They permit to block the degree of freedom along and around these axes and make the numerical simulation possible (as explained in Sect. 11.3).

\section{The Spherical Joints}

Eight spherical joints are present in the system: four between the SARIB beaters and the MGB bars and also four between the MGB bars and the MGB. The bond graph modeling of the spherical joint between the SARIB beater and an MGB Bar 


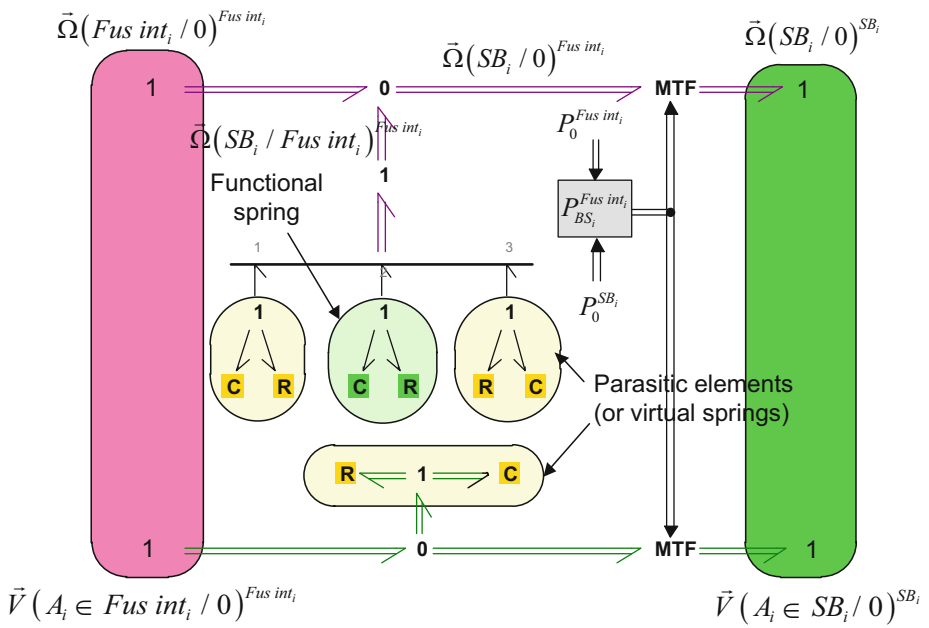

Fig. 11.22 Bond graph model of the revolute joint between the SARIB beaters and the fuselage

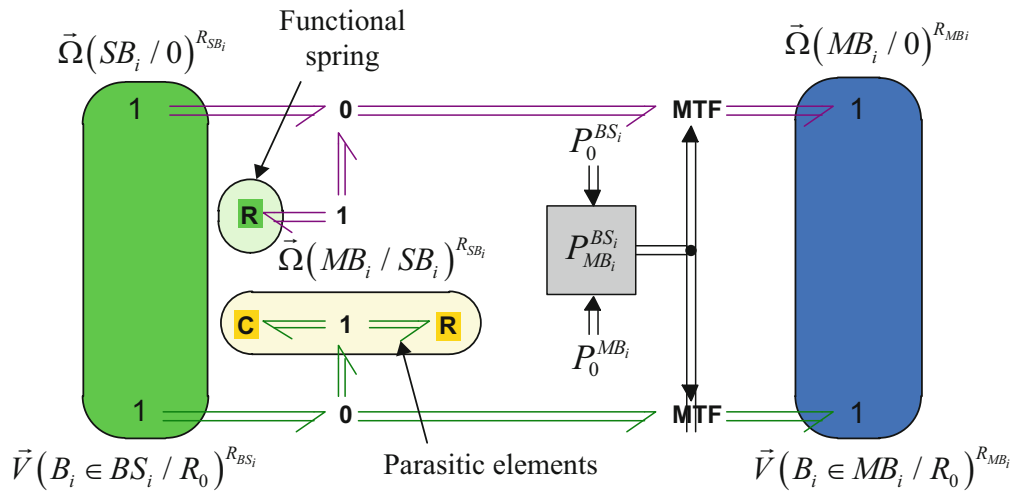

Fig. 11.23 Bond graph model of the spherical joint between an MGB bar $i$ and an SARIB beater $i$

is described in Fig. 11.23. The latter shows the blocking of the translational degree of freedom in which the relative translational velocities are close to zero, thanks to the virtual springs. The other spherical joints are built in the same manner.

\section{The Prismatic Joints}

The bond graph model of the prismatic joint between the MGB and an intermediate body is given in Fig. 11.24. 


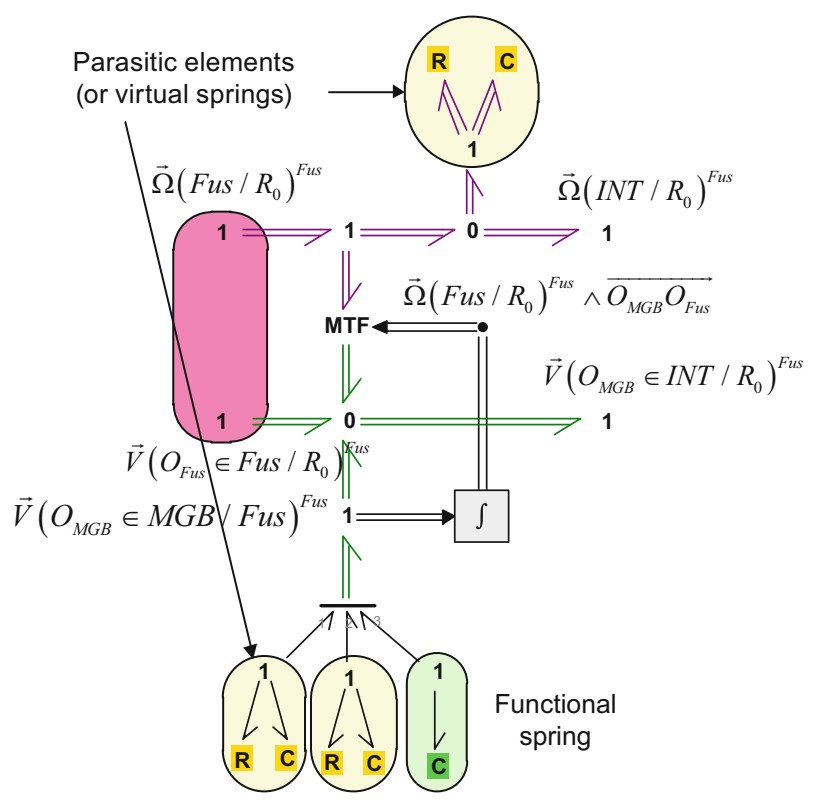

Fig. 11.24 Bond graph model of the prismatic joint between the MGB and an intermediate body

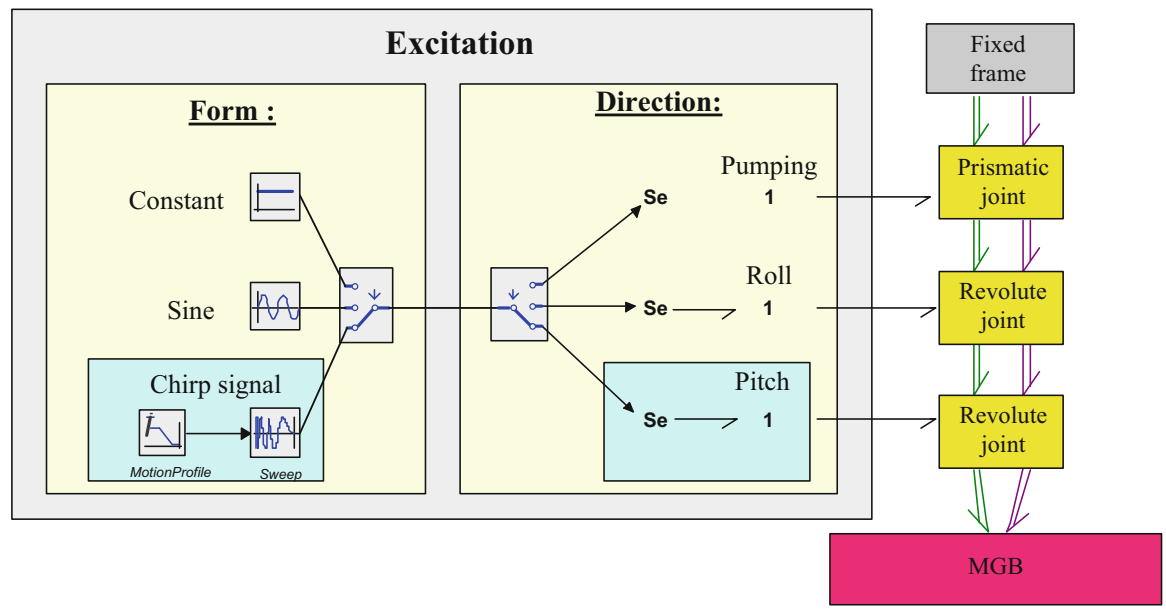

Fig. 11.25 Bond graph model of the actuators of the excitation

\subsubsection{Excitation Models}

The mechanical actions developed by the actuators are modeled by an effort source connected to the junctions $\mathbf{1}$ which are free in the attach joint. The example of the implementation of an excitation by a swept sine at the pitch joint is shown in Fig. 11.25. 
Depending on the selected excitation direction (pumping, roll, or pitch), this source will be, respectively, connected either to the free junction of the prismatic joint, or the revolute joints around the roll or pitch axis. Three choices of excitation forms may also be selected: constant for a natural mode excitation (if this constant is zero) or a static study, sinusoidal, or swept sine for excited modes.

The practical realization of these actuators will be conducted using vibration shakers as we will detail in the experimental validation section.

\subsubsection{Assembly}

These rigid bodies and joints models, as described above, are then connected together according to the architecture defined in the kinematic diagram. The bond graph model of the 3D MGB-fuselage suspension is given below in Fig. 11.26. As expected, the structural aspect of the model is explicit in so far as the structure of the model is similar to the joints graph of the system.

\subsubsection{Simulation Protocol}

\section{Getting Equations}

The step of generating the mechanical equations is fully automated and transparent to the user using the 20-sim software. In this step, the solver reads data corresponding to the bond graph model and builds a mathematical problem: the equations of the system.

\section{Resolution of the Equations}

The used bond graph software (20-sim) then solves the equations. With the method of singular perturbation, this step resolution can be performed simply using classical integration schemes for solving differential equations ODE solver such as Runge Kutta 4 (RK4). However, the integration scheme that has been used is the Backward Differentiation Formula (BDF). It was preferred as it allows a simulation of a stiff problem which is much faster than explicit solvers (like RK4). It is Gear's backward differentiation formula which is an implicit multi-step variable-order method. The implementation of the integrator is based on the Dassl library which has been developed in [10]. This simulation algorithm has required two parameters: the absolute and relative integration which has been tuned on the default values (absolute integration error $=\mathrm{e}-5$ and relative integration error $=\mathrm{e}-5$ ). This method chooses the step and the order in function of the error. Moreover, the BDF solver guarantees the numerical stability of the solution. From the mathematical solution, the solver computes and communicates the results requested by the user. 20-sim 


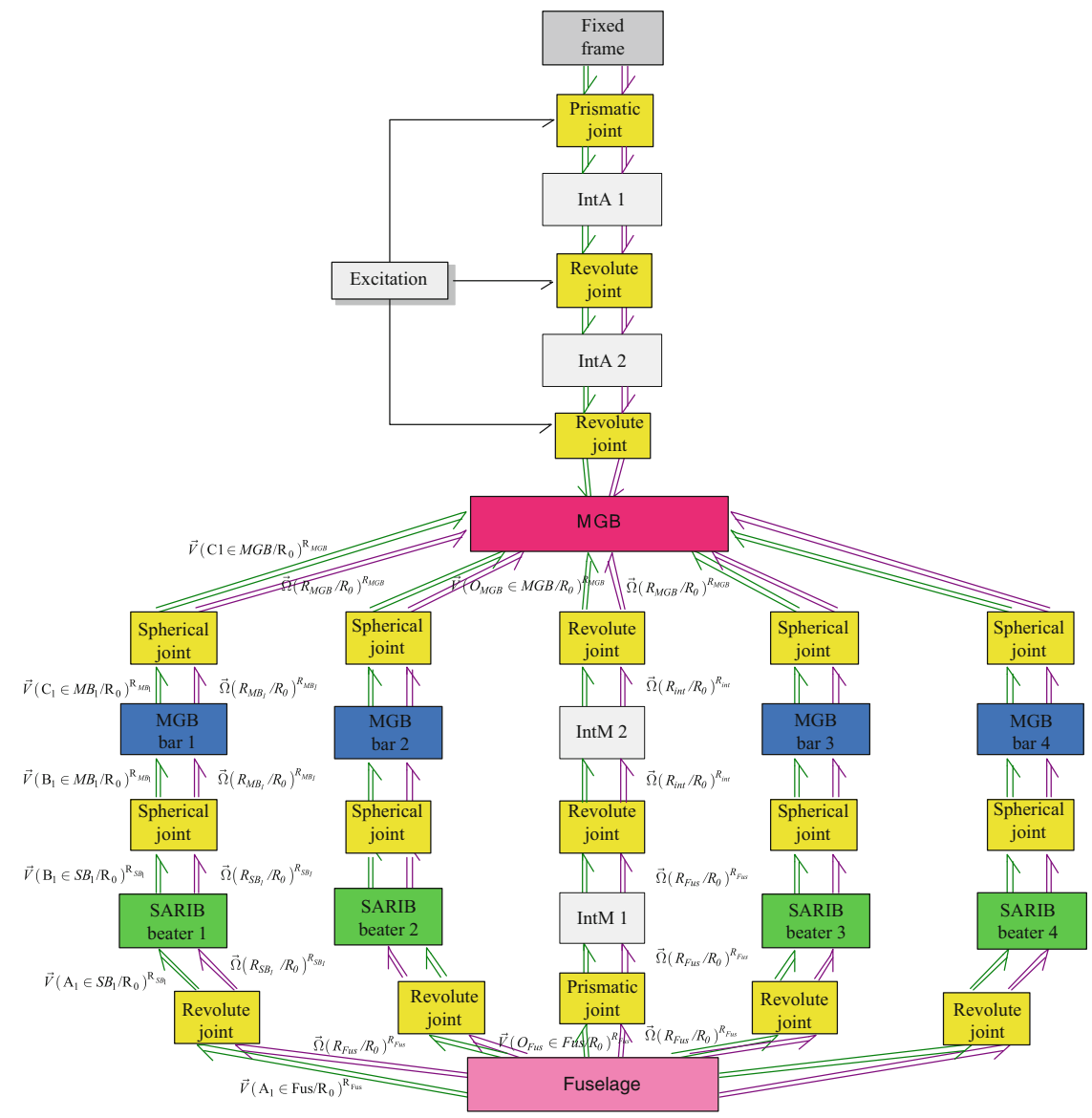

Fig. 11.26 Bond graph model of the passive MGB-fuselage suspension

allows the user to evaluate and easily plot any of the physical quantities involved in the bond graph model of the system.

\section{Post-Processing}

From the mathematical solution, the solver computes and communicates the results requested by the user. 20-sim allows the user to evaluate and easily plot any of the physical quantities involved in the bond graph model of the system. In addition, graphs can be exported to various types of image-formats or data with .csv or .xls file. The data exportation allows for the comparison of results from different sources in the same graphic interface (for example, Matlab). 


\subsection{Simulation and Verification of the Vibration Absorber Suspension Model}

\subsubsection{Use of MapleSim for BG Model Verification}

So as to verify the BG suspension model, simulation results conducted with MapleSim software (which contains a library dedicated to multibody systems) have been used. The simulation parameters used for both Bond graph conducted with 20-sim and MapleSim are summarized in Table 11.1.

\subsubsection{Comparison of Simulation Numerical Results}

The vibration analysis of the system is mainly evaluated by analyzing the acceleration of the fuselage points and forces transmitted from the MGB to the fuselage at the SARIB beater/ fuselage joint. Simulation results with different types of excitation (pumping, roll, and pitch) are the following and will permit to analyze the dynamic behavior of the suspension. The results presented were compared with a multibody software MapleSim.

\subsubsection{Dynamic Behavior with Pumping Excitation}

Acceleration of the Point $O_{F}$ of the Fuselage

The point $O_{F}$ is defined as the intersection of the MGB's axis and the upper plane of the fuselage. It is a central point on the fuselage that can be measured.

The vertical component of the acceleration of this point is given in Fig. 11.27

Table 11.1 Simulation comparison

\begin{tabular}{l|l|l}
\hline Software & MapleSim & $\begin{array}{l}\text { Bond graph conducted } \\
\text { with 20-sim }\end{array}$ \\
\hline Solver & Rosenbrock (stiff) & $\begin{array}{l}\text { Backward } \\
\text { differentiation } \\
\text { formula (BDF) }\end{array}$ \\
\hline Method & Linear graph theory & Singular perturbation \\
\hline $\begin{array}{l}\text { Number of } \\
\text { coordinates }\end{array}$ & 84 (14 bodies) & 84 (14 bodies) \\
\hline $\begin{array}{l}\text { Number of } \\
\text { constraints }\end{array}$ & $\begin{array}{l}74(8 \text { spherical joints, } \\
8 \text { revolute joints, } 2 \\
\text { prismatic joints })\end{array}$ & 0 \\
\hline DoF & $10(6+4)$ & 84 \\
\hline
\end{tabular}



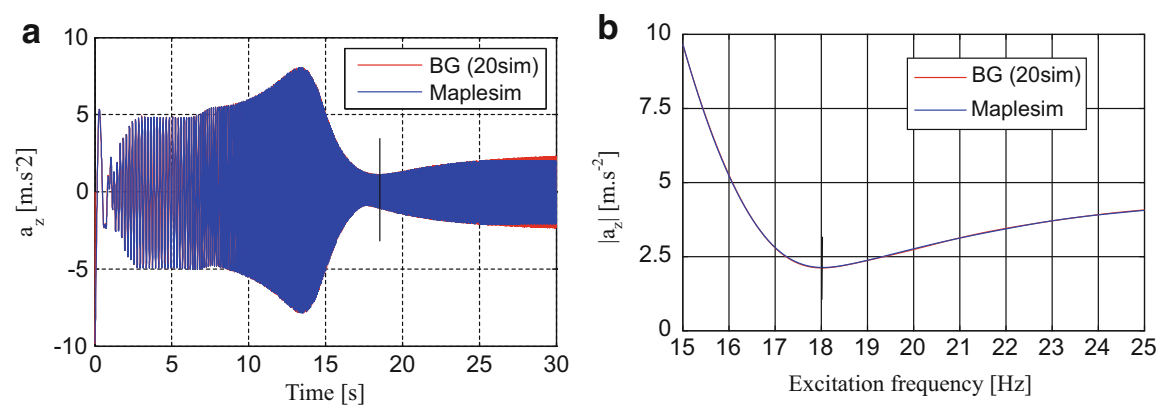

Fig. 11.27 Vertical component of the acceleration of the point $O_{F}$ for a pumping excitation (a) with chirp signal, (b) with sine signal
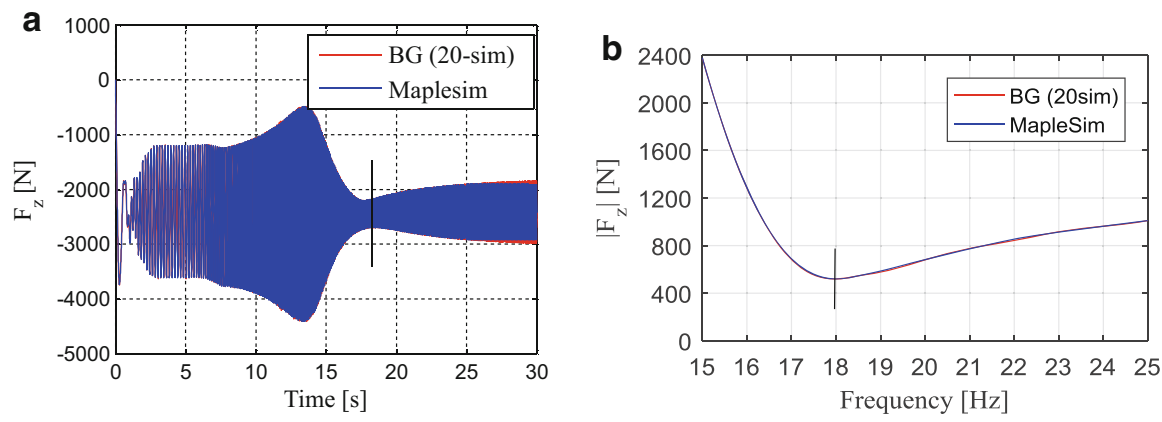

Fig. 11.28 Vertical force transmitted at the revolute joint A1 for a pumping excitation (a) with chirp signal, (b) with sine signal

An anti-resonance frequency is observed at $18 \mathrm{~Hz}$. The curve obtained with MapleSim is very close to the curve obtained with the simulation of the bond graph model with 20-sim.

\section{Forces Transmitted to the Fuselage}

The forces transmitted to the fuselage at the revolute joints between the SARIB beaters and the fuselage expressed in the intermediate pins fuselage were determined as shown in Fig. 11.28.

As shown in the above figures, the curves of the vertical forces transmitted at the revolute joints are logically similar to the curve of the acceleration of the point $O_{F}$. Through the action of inertial forces, the transmission forces of the MGB to the fuselage are reduced at a certain frequency called anti-resonance frequency. They present an anti-resonance at about $18 \mathrm{~Hz}$. SARIB beaters thus play their roles.

We can observe in Fig. 11.29 that the anti-resonance phenomenon observed in the force along $x$-axis in the intermediate fuselage frames does not occur at the same 

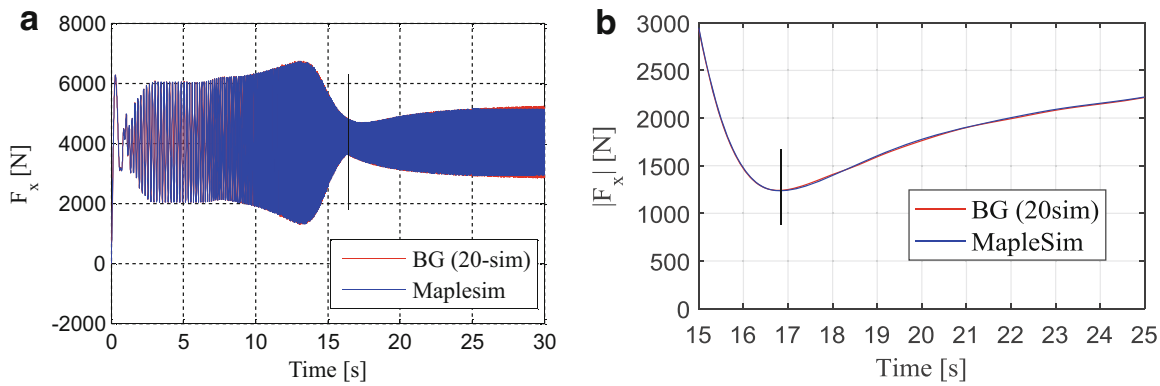

Fig. 11.29 Force along $x$-axis in the intermediate fuselage frame at the revolute joint A1 for a pumping excitation (a) with chirp signal, (b) with sine signal
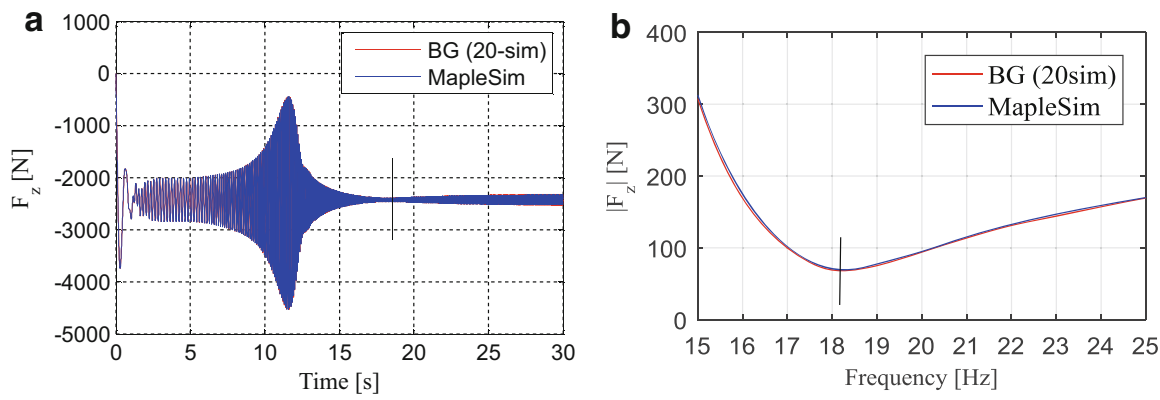

Fig. 11.30 Vertical force at the revolute joint A1 for a roll excitation (a) with chirp signal, (b) with sine signal

frequency as the one which had been observed for the transmission of vertical forces to the fuselage (for reminding around $18 \mathrm{~Hz}$ ). The frequency of anti-resonance is around $16.8 \mathrm{~Hz}$. The designer will have to monitor the amplitude of the component along $x$-axis of these forces which are not minimal at the anti-resonant frequency for the vertical forces transmitted to the fuselage.

\subsubsection{Dynamic Behavior with Roll Excitation}

The vertical force transmitted to the fuselage at the revolute joint A1 between the SARIB beaters and the fuselage expressed in the intermediate fuselage frames was determined as shown in Fig. 11.30. As previously, an anti-resonance phenomenon is observed at the frequency of $18.2 \mathrm{~Hz}$.

In Fig. 11.31, we can notice the phase shift between the vertical force at the revolute joint $\mathrm{A} 1$ and the revolute joint $\mathrm{A} 2$ when the MGB is submitted to a roll excitation. 

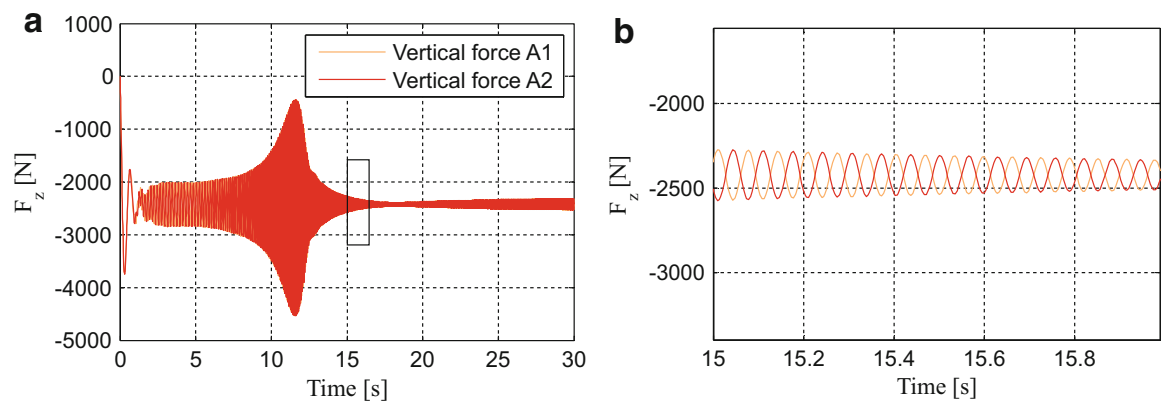

Fig. 11.31 (a) Vertical forces at the revolute joints A1 and A2 with a roll excitation, (b) zoom

\subsubsection{Dynamic Behavior with Pitch Excitation}

Similar results are observed for a pitch excitation but are not presented for the sake of concision.

\subsubsection{About the Modeling and Simulation of a Semi-Active Suspension}

In the frame of this research work, a semi-active suspension has also been modeled and simulated. The tuning of the anti-resonant frequency is then obtained by changing the position of the moving masses on SARIB beaters. The objective of the semi-active suspension is to improve the reduction of vibrations despite some fluctuations of the frequency of the rotor excitation by maintaining the positioning of the anti-resonant frequency of the suspension on the rotor excitation frequency. For this purpose, the position of the moving masses driven by electric DC motors and screw-nut systems has been optimized and PI controllers have permitted to position the moving masses to the setpoints generated by a control algorithm. Models and simulations on this semi-active suspension model have been conducted in [8] and are not here developed for the sake of concision. This study shows that multiphysic properties of the bond graph approach enable to add easily electrical actuators and a control command structure directly in a unique model.

\subsection{Conclusion}

A structural and modular approach with bond graphs has been presented in this chapter so as to model and simulate the dynamics of a complex mechatronic multibody system: a helicopter's vibration absorber system. 
Three main methods (ZCP, R/C elements, and Lagrange Multipliers) of simulating BG models of multibody systems have been presented. Both the conditions of application and practical rules for applying these methods with graphical bond graph software, such as 20-sim software, have been given.

The proposed methodology consists of the assembly of modules (these components are rigid bodies and compliant kinematic joints) in a similar manner to the real structure of the physical system. This assembly can be easily conducted with the help of a well-structured library of components. Therefore, the constructed multibody dynamic model permits the representation of a complex system and the bond graph model highlights the topology of systems.

The parasitic compliant elements in the kinematic joints have been used to avoid dealing with kinematic constraint equations and consequently to have only ODE systems to solve instead of DAEs. Moreover, this method has suppressed the constraints linked to the causality imposed by the use of vector bonds.

The simulation results of the bond graph model of the studied suspension have been conducted with dedicated software for bond graphs such as 20-sim and have been verified with another multibody tool (MapleSim) for three excitations (pumping, roll, and yaw). These simulations have permitted the identification and quantification of the anti-resonance which were sought so as to filter the vibrations coming from the rotor to the fuselage.

It has been shown than the BG should permit a structural and modular modeling of complex 3D mechatronic systems. It is worthwhile to note that the most recent structural commercial modeling tools such as MapleSim and others could also be used for the modeling of the studied suspension. However, to take benefit from the unified power-based approach and exploit specific properties of BG, bond graphs have been chosen as the modeling language. Future works are being conducted so as to exploit two promising BG exploitations which are the analysis of energetic flow transiting among components and the control of multibody systems by means of inversion techniques permitted by BG structural analysis [21].

Acknowledgments This research work received support from the Chair "Dynamics of complex mechanical systems - EADS Corporate Foundation - Arts et Métiers ParisTech and Ecole Centrale de Marseille." Special thanks to Paul B.T. Weustink who works at Controllab Products for his help with the use of complementary tools from 20-sim software.

\section{References}

1. Abadie, V., Guillemard, F., \& Rault, A. (2000). Apport du bond graph dans la démarche mécatronique appliquée à l'automobile. In G. Dauphin-Tanguy (Ed.), Les bond graphs. Paris: Hermès Sciences Europe Ltd. ISBN:2-7462-0158-5.

2. Amerongen, J. V., \& Breedveld, P. (2003). Modelling of physical systems for the design and control of mechatronic systems. Annual Reviews in Control, 27(1), 87-117.

3. Behzadipour, S., \& Khajepour, A. (2005). Causality in vector bond graphs and its application to modeling of multi-body dynamic systems. Simulation Modelling Practice and Theory, 14, 279-295. 
4. Bonderson, L. S. (1975). Vector bond graphs applied to one-dimensional distributed systems. Journal of Dynamic Systems, Measurement, and Control, 1, 75-82.

5. Borutsky, W. (2010). Bond graph methodology-Development and analysis of multidisciplinary dynamic system models (Springer ed.th ed.). London: Springer.

6. Borutzky, W. (1995). Representing discontinuities by means of sinks of fixed causality. In: Proceedings of the 1995 Western Simulation Multiconference, Las Vegas, Nevada.

7. Bos, A. M. (1986). Multibody systems in terms of multibond graphs with application to a motorcycle multibody system. $\mathrm{PhD}$ thesis, University of Twente, Enschede, The Netherlands.

8. Boudon, B., Malburet, F., \& Carmona, J. C. (2016). Simulation of a helicopter's main gearbox semiactive suspension with bond graphs. Multibody System Dynamics, 1-31.

9. Breedveld, P. C. (1985). Multibond graph elements in physical systems theory. Journal of the Franklin Institute, 319, 1-36.

10. Brenan, K. E., Campbell, S. L., \& Petzold, L. R. (1996). Numerical solution of initial-value problems in differential-algebraic equations. SIAM. ISBN:978-0-89871-353-4.

11. Cacho, R., Felez, J., \& Vera, C. (2000). Deriving simulation models from bond graphs with algebraic loops: The extension to multibond graph systems. Journal of the Franklin Institute, $337,579-600$.

12. Ersal, T., Stein, J. L., \& Louca, L. S. (2004). A bond graph based modular modeling approach towards an automated modeling environment for reconfigurable machine tools. In International conference on integrated modeling and analysis in applied control and automation (2004).

13. Felez, J., Romero, G., Maroto, J., \& Martinez, M. L. (2011). Simulation of multi-body systems using multi-bond graph. In W. Borutsky (Ed.), Bond graph modeling of engineering systems. Springer. ISBN:978-1-4419-9367-0.

14. Felez, J., Vera, C., San Jose, I., \& Cacho, R. (1990). BONDYN: A bond graph based simulation program for multibody systems. Journal of Dynamic Systems, Measurement, and Control, 112, 717-727.

15. Henderson, J.-P. (2012). Vibration isolation for rotorcraft using electrical actuation. $\mathrm{PhD}$ thesis, Mechanical Engineering, University of Bath.

16. Karnopp, D. (1969). Power-conserving transformations: Physical interpretations and applications using bond graphs. Journal of the Franklin Institute, 288, 175-201.

17. Karnopp, D. C., \& Margolis, D. L. (1979). Analysis and simulation of planar mechanisms systems using bond graphs. Journal of Mechanical Design, Transactions of the ASME, 101, 187-191.

18. Krysinski, T., \& Malburet, F. (2006). Mechanical vibrations: Active and passive control. London: Wiley-ISTE.

19. Lalanne, C. (2014). Mechanical vibration and shock analysis. London: Wiley-ISTE.

20. Marquis-Favre, W. (1997). Contribution à la représentation bond graph des systèmes mécaniques multicorps. PhD thesis, INSA de Lyon.

21. Marquis-Favre, W., \& Jardin, A. (2011). Bond graphs and inverse modeling for mechatronic system design. In: W. Borutsky (Ed.), Bond graph modeling of engineering systems. Berlin: Springer.

22. Marquis-Favre, W., \& Scavarda, S. (1998). Bond graph representation of multibody systems with kinematic loops. Journal of the Franklin Institute, 335B, 643-660.

23. Paynter, H. M. (1961). Analysis and design of engineering systems. Cambridge: MIT Press.

24. Rideout, G. (2004). System partitioning and physical domain proper modeling through assessment of power-conserving model structure. $\mathrm{PhD}$ thesis, University of Michigan.

25. Romero, G., Felez, J., Maroto, J., \& Cabanellas, J. M. (2007). A minimal set of dynamic equations in systems modelled with bond graphs. Proceedings of the Institution of Mechanical Engineers, Part I: Journal of Systems and Control Engineering, 221(1), 15-26.

26. Rosenberg, R. C. (1972). Multiport models in mechanics. Journal of Dynamic Systems, Measurement, and Control, 94, 206-212. 
27. Sass, L., McPhee, J., Schmitke, C., Fisette, P., \& Grenier, D. (2004). A comparison of different methods for modelling electromechanical multibody systems. Multibody System Dynamics, 12, 209-250.

28. Schiehlen, W. (2007). Research trends in multibody system dynamics. Multibody System Dynamics, 18, 3-13.

29. Tiernego, M. J. L., \& Bos, A. M. (1985). Modelling the dynamics and kinematics of mechanical systems with multibond graphs. Journal of the Franklin Institute, 319, 37-50.

30. van Dijk, J., \& Breedveld, P. C. (1991). Simulation of system models containing zero-order causal paths-I. Classification of zero-order causal paths. Journal of the Franklin Institute, 328, 959-979.

31. van Dijk, J., \& Breedveld, P. C. (1991). Simulation of system models containing zero-order causal paths-II Numerical implications of class 1 zero-order causal paths. Journal of the Franklin Institute, 328, 981-1004.

32. Wang, J., Gosselin, C., \& Cheng, L. (2001). Modeling and simulation of robotic systems with closed kinematic chains using the virtual spring approach. Multibody System Dynamics, 7, $145-170$.

33. Xu, W., Liu, Y., Liang, B., Wang, X., \& Xu, Y. (2010). Unified multi-domain modelling and simulation of space robot for capturing a moving target. Multibody System Dynamics, 23, 293-331.

34. Zeid, A., \& Chung, C.-H. (1992). Bond graph modeling of multibody systems: a library of three-dimensional joints. Journal of the Franklin Institute, 329, 605-636.

35. Zeid, A., \& Overholt, J. L. (1995). Singularly perturbed formulation: Explicit modeling of multibody systems. Journal of the Franklin Institute, 332. 